\title{
Buckling Imperfection Sensitivity of Axially Compressed Orthotropic Cylinders
}

\author{
Marc R. Schultz ${ }^{1}$ and Michael P. Nemeth ${ }^{2}$ \\ NASA Langley Research Center, Hampton, VA 23681-2199, USA
}

\begin{abstract}
Structural stability is a major consideration in the design of lightweight shell structures. However, the theoretical predictions of geometrically perfect structures often considerably over predict the buckling loads of inherently imperfect real structures. It is reasonably well understood how the shell geometry affects the imperfection sensitivity of axially compressed cylindrical shells; however, the effects of shell anisotropy on the imperfection sensitivity is less well understood. In the present paper, the development of an analytical model for assessing the imperfection sensitivity of axially compressed orthotropic cylinders is discussed. Results from the analytical model for four shell designs are compared with those from a general-purpose finite-element code, and good qualitative agreement is found. Reasons for discrepancies are discussed, and potential design implications of this line of research are discussed.
\end{abstract}

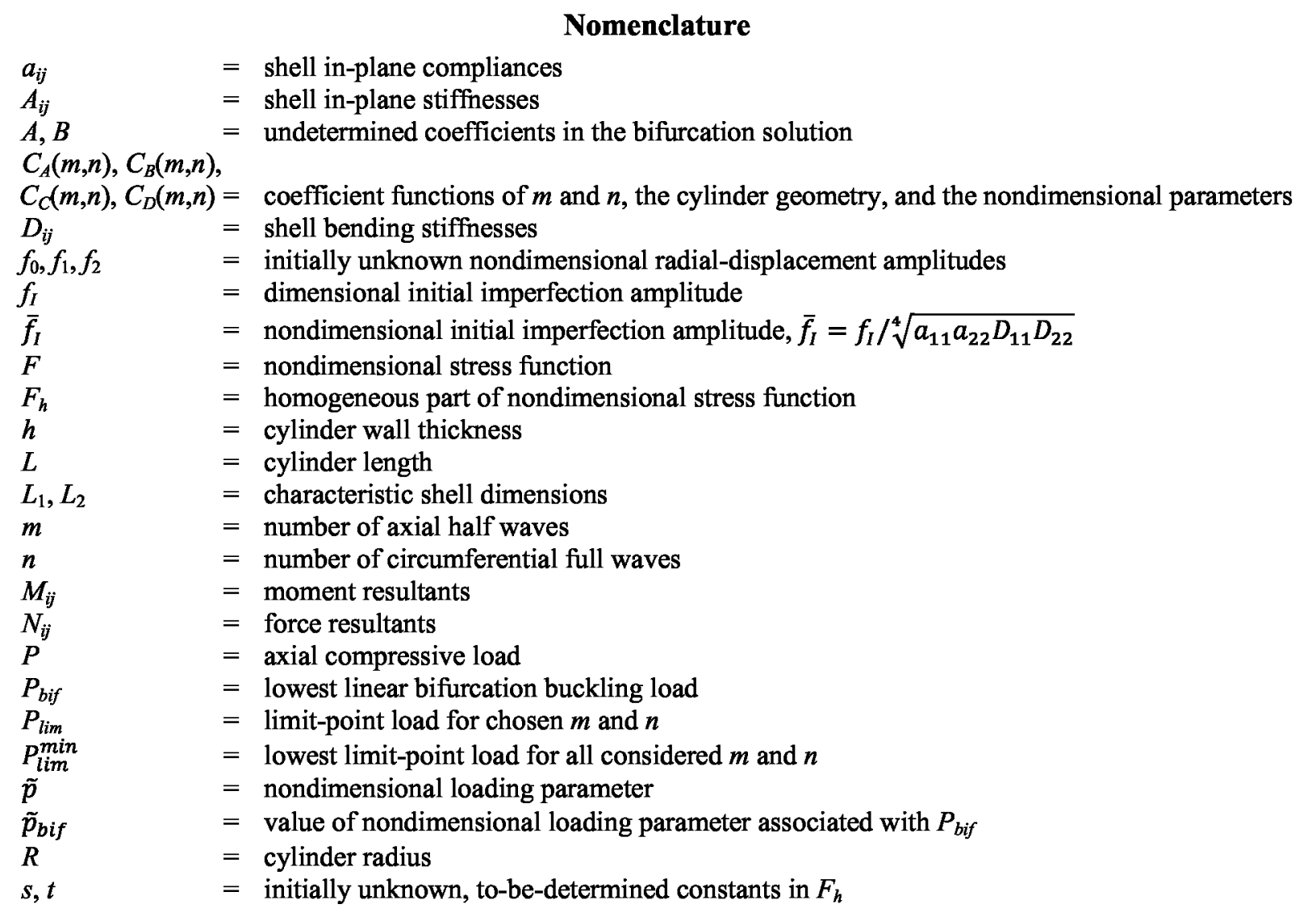

${ }^{1}$ Aerospace Engineer, Structural Mechanics and Concepts Branch, 8 West Taylor St., Mail Stop 190, AIAA Senior Member.

${ }^{2}$ Senior Research Engineer, Structural Mechanics and Concepts Branch, 8 West Taylor St., Mail Stop 190, AIAA Associate Fellow. 


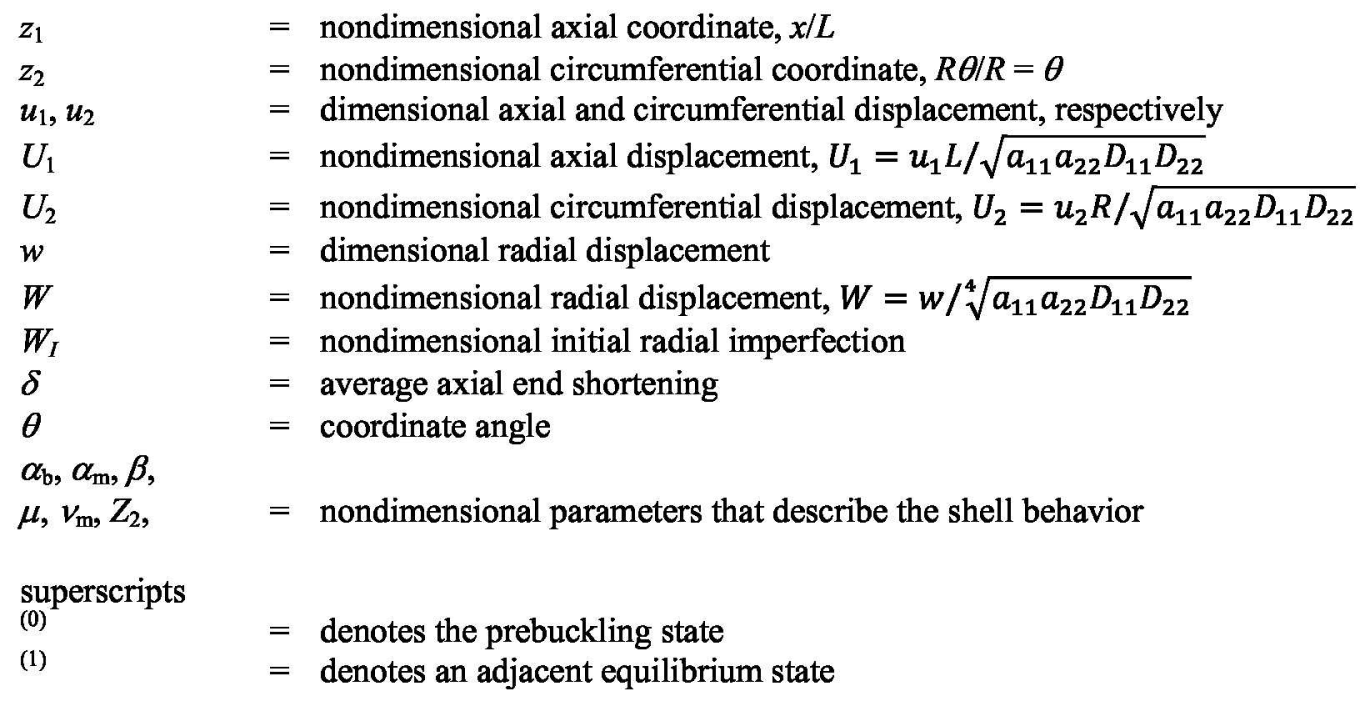

\section{Introduction}

tructural stability is a major consideration in the design of lightweight structures, and is often predicted by using relatively simple linear eigenvalue buckling analyses that are based on idealized, perfect geometry. During the past one hundred years, a large collection of theoretical buckling predictions for many idealized structures under different loading conditions has been developed. However, these classical theoretical predictions are often considerably nonconservative for certain classes of inherently imperfect thin-walled structures, such as cylinders. To account for this nonconservatism in a design, a correction factor known as the buckling knockdown factor is often used to lower the theoretical predictions to a safe level.

Though all real structures are imperfect, different structural forms and constructions have different sensitivity to imperfections. In particular, the imperfection sensitivity of a structure is influenced by the geometry, construction, material properties, and the support and loading conditions. In order to partially explain the nature of imperfection sensitivity, two notional axial load versus end-shortening response curves for structures with relatively small geometric imperfections are shown in Fig. 1. For brevity, these curves are referred to herein as load-response curves. Figure 1a shows the behavior of a typical imperfectionsensitive cylindrical shell, and Fig. $1 \mathrm{~b}$ shows the behavior of a typical flat plate. In each of these figures, the theoretical behavior of the idealized perfect structure is shown with a dashed line, and the behavior of the corresponding imperfect structure is shown with a solid line. The loadresponse curve of the perfect, highly imperfectionsensitive cylinder (Fig. 1a) is characterized by a steep, narrow cusp with large drop in load after the maximum load, $P_{b i f}$, is reached. For this case, the maximum load occurs at a bifurcation point (eigenvalue) where unstable equilibrium states that are adjacent to the primary, linear equilibrium path exist. The figure also shows that the load-response

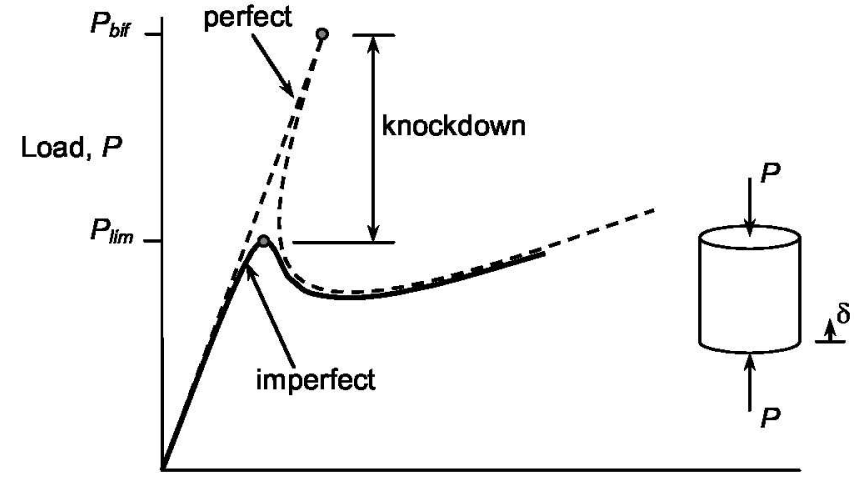

End Shortening, $\delta$

(a) Moderately long cylinder

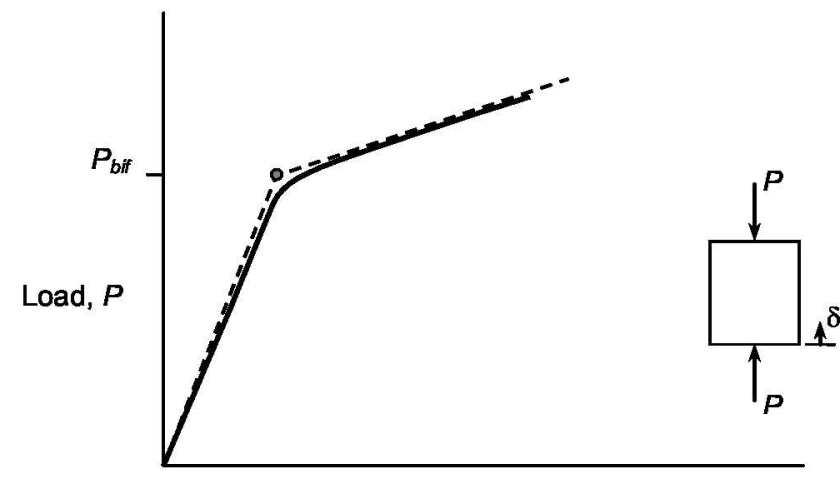

End Shortening, $\delta$

(b) Flat plate

Figure 1. Notional load vs. end shortening curves for geometrically perfect and imperfect structures.

2

American Institute of Aeronautics and Astronautics 
curve for the imperfect cylinder only extends part way into the cusp and exhibits a maximum, limit-point load, $P_{\text {lim }}$, that is significantly lower than $P_{b i f}$. The difference in these two buckling loads has been substantiated by numerous experiments. ${ }^{1}$ Thus, the predicted bifurcation-point load is not an adequate measure of the actual buckling resistance, and buckling knockdown factors are needed to ensure that this imperfection sensitivity is taken into account when classical analysis methods are used to design a shell. To illustrate how geometry affects imperfection sensitivity, the load-response curve for a flat plate is shown in Fig. 1b. The bilinear dashed line shows a loss of stiffness but no sudden loss of load carrying capacity when the buckling load, $P_{b i f}$, is reached. For this case, $P_{b i f}$ represents a bifurcation point (eigenvalue) where stable equilibrium states that are adjacent to the primary, linear equilibrium path exist. The lack of a cusp, associated with an unstable bifurcation, and the proximity of the load level where a significant stiffness change occurs for the imperfect plate indicates that the plate is not sensitive to relatively small imperfections. Thus, the classical bifurcation-point load is an adequate measure of the buckling resistance when designing structures that display this type of behavior.

In the 1960s, NASA developed shell stability design recommendations that were published as a series of monographs. The best known is NASA SP- $8007^{1}$ which gives design recommendations and knockdown factors for thin-walled cylindrical shells; isotropic unstiffened, orthotropically stiffened, isotropic sandwich, and elastic-cored cylinders are considered. Others monographs ${ }^{2,3}$ deal with conical and doubly curved shells. Though these documents are widely used, they are based on test data, computational methods, and resources of the 1930-1960's and are thought to be overly conservative. ${ }^{4,5}$ Additionally, few data for laminated-composite shells were available when these recommendations were developed and, and as a result, many designers arbitrarily apply the knockdown factors for isotropic monocoque, stiffened, and sandwich shells to laminated-composite shells, for lack of anything better. In many cases, the misapplication of the knockdown factor also results in excessive conservatism. This excessive conservatism usually corresponds to an increase in structural mass, which is particularly important in the development of launch vehicles. Thus, improved knockdown factors that can be used to design the acreage regions of shell structures can produce significant mass savings in large launch-vehicle structures such as tanks, intertanks, interstages, boosters, and shrouds.

Since NASA's shell-buckling monographs were written, there have been many improvements made to analytical tools, and experimental and measurement techniques. ${ }^{4}$ Of particular interest to the shell buckling problem are the advancement and wide-spread use of finite-element methods, and measurement techniques that allow accurate measurement of actual as-manufactured shell geometries and real-time response. In order to make use of these and other advancements, efforts are underway to develop recommendations for improved knockdown factors that are conservative, but not excessively conservative. One general approach is to develop a characteristic geometric imperfection "signature" associated with a particular manufacturing process that can be used with modern analysis techniques to develop refined buckling knockdown factors. This type of approach has been outlined by Hilburger, et $\mathrm{al}^{5}$ and Arbocz and $\mathrm{Hol}^{6}$ for laboratory-scale specimens.

Even if new methods for determining the knockdown factors based on known characteristic geometric imperfection signatures are developed, it would be helpful for designers to know a priori which constructions are likely to be more sensitive to imperfections. With this knowledge, judgments can be more easily made regarding the need for more costly analysis methods or experiments, and over time, the institutional dependence on empiricism can be reduced. Although a number of authors have explored the imperfection sensitivity of nonisotropic cylinders (see for example, refs. 5-11), it appears that there have been no efforts to develop a general understanding of how orthotropy and anisotropy affect the imperfection sensitivity of compression-loaded cylinders. If industry is to take full advantage of the potential mass savings that composite materials and improved knockdown factors offer, a general understanding of how orthotropy and anisotropy affect imperfection sensitivity is required.

Two common approaches to developing a broad understanding of imperfection sensitivity would be to use a general-purpose finite-element code or to develop special-purpose analyses tailored to a specific class of problems that are relevant to design. Using a finite-element code has the advantages that it is readily available and can handle many different geometries and loading conditions in a robust manner. However, each analysis can take a long time, the model must be recreated each time geometry changes, and little insight into the problem is gained from the model formulation. Moreover, additional convergence studies may be required as problem variables change. Collectively, these attributes of general-purpose finite-element codes make rapid navigation of design spaces tedious. In contrast, a special-purpose analysis typically runs much faster than a corresponding finite-element analysis done with a general-purpose code, and is inherently amenable to parametric studies because it easily accommodates changes in shell-wall construction and geometry. However, the inherent simplifications present in a special-purpose analysis are manifested by a limited range of validity that must be established within the context of the fidelity required for design. 
The goal of the current study is to present the first steps in the development of a first-approximation special-purpose analysis tool for obtaining conservative estimates of the imperfection sensitivity of compression-loaded orthotropic cylinders. The analysis is formulated directly in terms of nondimensional parameters that characterize the design space adequately. Nondimensional parameters, such as the ones used herein, are significant in that they often provide insight into similar response characteristics and trends, and provide a means for rapid navigation through the very large design space of laminated-composite cylinders. Additionally, nondimensional parameters can provide a means for reducing the effort involved in establishing the range of validity of the analysis by reducing the number of design parameters. In most design settings, measured imperfections do not yet exist and an alternative means for simulating their effects is needed. In the present study, bifurcation buckling modes are used to represent initial geometric-imperfection shapes, and

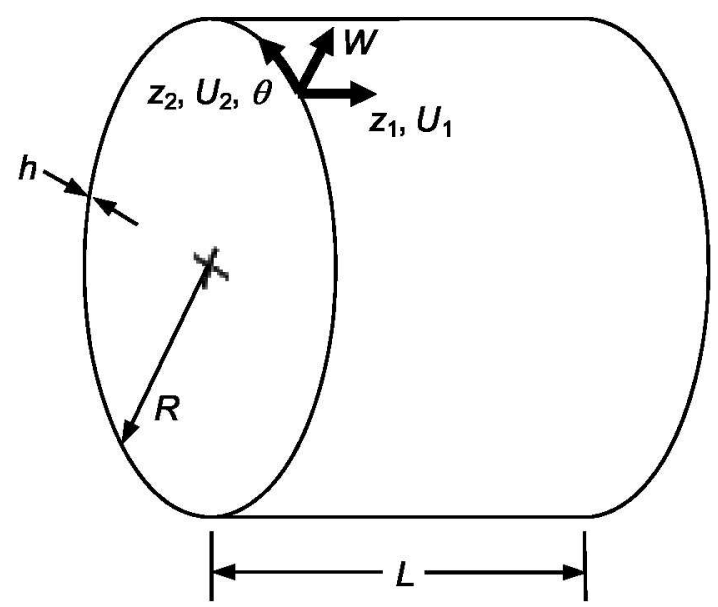

Figure 2. Geometry, coordinate system, and mid-surface displacements. are referred to herein as modal imperfections. A full range of modal imperfections are used in the analyses to find the modal imperfection that gives the lowest limit-point load for a given shell construction and imperfection amplitude. The results are expected to be conservative because modal imperfections represent deformation shapes with a high bias toward buckling.

To accomplish the goal of the present study, the development of the analytical model is discussed first. Then, results from the analytical model for an isotropic monocoque cylinder are described in detail and the results are compared with results from finite-element analyses. These results demonstrate all facets of the analytical solution process and establish the logic behind obtaining imperfection-sensitivity estimates. Next, results from the analytical model are presented for three different composite sandwich cylinders that are representative of heavy-lift launch vehicle structures, and the results are compared with finite-element analyses. Finally, the design implications of this line of research are discussed.

\section{Analytical Model Development}

There is a significant body of literature in which the buckling of cylindrical shells and the effects of geometric imperfections on the buckling behavior are examined. ${ }^{5-18}$ In this paper, the nondimensional parameters and equations of Refs. 19 and 20, based on Donnell's equations, ${ }^{12,14,15}$ are applied to axially loaded, geometrically imperfect, closed cylindrical shells with the geometry and coordinate system shown in Fig. 2 . More specifically, the analysis presented in this section uses the stress-function formulation presented in Refs. 19 and 20. Though Donnell's equations are often referred to as shallow-shell equations, they are valid for closed shells, provided that the deformations have more than approximately three circumferential waves. ${ }^{15}$ Additionally, Donnell's equations neglect transverse shear flexibility, which has the practical effect of limiting the validity of the equations to thin shells - for cylinders, those with large radius/thickness $(R / h)$ ratios. For example, for isotropic cylindrical shells, it is generally agreed that $R / h>20$ is required to use Donnell's equations. For composite shells, particularly sandwich shells, larger values of this ratio may required because composites often have relatively low transverse shear stiffness.

\section{A. Bifurcation Buckling of Perfect Cylinders}

The equations used herein to determine the bifurcation buckling load and the corresponding modes are obtained by specializing the equations given in Ref. 20 for a generally anisotropic doubly curved shell to the case of an axialcompression-loaded orthotropic cylinder. This simplification is accomplished by setting the constitutive constants $A_{16}=A_{26}=D_{16}=D_{26}=0$ in addition to the constants associated with coupling between bending, twisting, extension, and shearing. Moreover, the characteristic dimensions used in Ref. 20 are specified as $L_{1}=L$ and $L_{2}=R$ (see Fig. 2). Applying these simplifications, the transverse equilibrium equation becomes 


$$
\begin{aligned}
& \alpha_{b}^{2} \frac{\partial^{4} W^{(1)}}{\partial z_{1}^{4}}+2 \beta \frac{\partial^{4} W^{(1)}}{\partial z_{1}^{2} \partial z_{2}^{2}}+\frac{1}{\alpha_{b}^{2}} \frac{\partial^{4} W^{(1)}}{\partial z_{2}^{4}}+\sqrt{12} Z_{2} \frac{\partial^{2} F^{(1)}}{\partial z_{1}^{2}}= \\
& \tilde{p} \frac{N_{11}^{(0)} R^{2}}{\sqrt{D_{11} D_{22}}} \frac{\partial^{2} W^{(1)}}{\partial z_{1}^{2}}+\frac{\partial^{2} F^{(1)}}{\partial z_{2}^{2}} \frac{\partial^{2} W^{(0)}}{\partial z_{1}^{2}}+\frac{\partial^{2} F^{(1)}}{\partial z_{1}^{2}} \frac{\partial^{2} W^{(0)}}{\partial z_{2}^{2}}-2 \frac{\partial^{2} F^{(1)}}{\partial z_{1} \partial z_{2}} \frac{\partial^{2} W^{(0)}}{\partial z_{1} \partial z_{2}}
\end{aligned}
$$

and the compatibility equation becomes

$$
\alpha_{m}^{2} \frac{\partial^{4} F^{(1)}}{\partial z_{1}^{4}}+2 \mu \frac{\partial^{4} F^{(1)}}{\partial z_{1}^{2} \partial z_{2}^{2}}+\frac{1}{\alpha_{m}^{2}} \frac{\partial^{4} F^{(1)}}{\partial z_{2}^{4}}-\sqrt{12} Z_{2} \frac{\partial^{2} W^{(1)}}{\partial z_{1}^{2}}=\frac{\partial^{2} W^{(0)}}{\partial z_{2}^{2}} \frac{\partial^{2} W^{(1)}}{\partial z_{1}^{2}}+\frac{\partial^{2} W^{(0)}}{\partial z_{1}^{2}} \frac{\partial^{2} W^{(1)}}{\partial z_{2}^{2}}-2 \frac{\partial^{2} W^{(0)}}{\partial z_{1} \partial z_{2}} \frac{\partial^{2} W^{(1)}}{\partial z_{1} \partial z_{2}}
$$

where $W^{(0)}$ and $W^{(1)}$ are the nondimensional radial displacements associated with the prebuckling and adjacent equilibrium states, respectively; $\tilde{p}$ is a nondimensional loading parameter; $N_{11}^{(0)}$ is the constant-valued axial stress resultant in the prebuckling state, $D_{11}$ and $D_{22}$ are the axial and circumferential bending stiffnesses, respectively; and $F^{(1)}$ is a nondimensional stress function related to the adjacent equilibrium states and identically satisfies the corresponding tangential equilibrium equations. For the cylinder, this stress function is defined as

$$
\begin{aligned}
& \frac{\partial^{2} F^{(1)}}{\partial z_{2}^{2}}=\frac{N_{11}^{(1)} R^{2}}{\sqrt{D_{11} D_{22}}} \\
& \frac{\partial^{2} F^{(1)}}{\partial z_{1}^{2}}=\frac{N_{22}^{(1)} L^{2}}{\sqrt{D_{11} D_{22}}} \\
& \frac{\partial F^{(1)}}{\partial z_{1} \partial z_{2}}=\frac{N_{12}^{(1)} R L}{\sqrt{D_{11} D_{22}}}
\end{aligned}
$$

The nondimensional parameters, $\alpha_{m}, \alpha_{b}, \beta, \mu$, and $\mathrm{Z}_{2}$, simplified for the cylinders considered herein are given by

$$
\begin{gathered}
\alpha_{m}=\frac{R}{L} \sqrt[4]{\frac{A_{11}}{A_{22}}} \\
\alpha_{b}=\frac{R}{L} \sqrt[4]{\frac{D_{11}}{D_{22}}} \\
\mu=\frac{A_{11} A_{22}-A_{12}^{2}-2 A_{12} A_{66}}{2 A_{11} \sqrt{A_{11} A_{22}}} \\
\beta=\frac{D_{12}+2 D_{66}}{\sqrt{D_{11} D_{22}}} \\
Z_{2}=\frac{R}{h} \sqrt{\frac{\left(A_{11} A_{22}-A_{12}^{2}\right) h^{2}}{12 \sqrt{A_{11} A_{22} D_{11} D_{22}}}}
\end{gathered}
$$

where $A_{11}, A_{12}, A_{22}$, and $A_{66}$ are the membrane stiffnesses, and $D_{12}$ and $D_{66}$ are additional bending stiffnesses. The symbol $Z_{2}$ denotes a stiffness-weighted thinness parameter. Next, the prebuckling bending deformations associated with the derivatives of $W^{(0)}$ are presumed negligible, consistent with a classical bifurcation-buckling analysis. With these simplifications, Eqs. (1) and (2) reduce to

$$
\alpha_{b}^{2} \frac{\partial^{4} W^{(1)}}{\partial z_{1}^{4}}+2 \beta \frac{\partial^{4} W^{(1)}}{\partial z_{1}^{2} \partial z_{2}^{2}}+\frac{1}{\alpha_{b}^{2}} \frac{\partial^{4} W^{(1)}}{\partial z_{2}^{4}}+\sqrt{12} Z_{2} \frac{\partial^{2} F^{(1)}}{\partial z_{1}^{2}}=\tilde{p} \frac{N_{11}^{(0)} R^{2}}{\sqrt{D_{11} D_{22}}} \frac{\partial^{2} W^{(1)}}{\partial z_{1}^{2}}
$$

and

$$
\alpha_{m}^{2} \frac{\partial^{4} F^{(1)}}{\partial z_{1}^{4}}+2 \mu \frac{\partial^{4} F^{(1)}}{\partial z_{1}^{2} \partial z_{2}^{2}}+\frac{1}{\alpha_{m}^{2}} \frac{\partial^{4} F^{(1)}}{\partial z_{2}^{4}}=\sqrt{12} Z_{2} \frac{\partial^{2} W^{(1)}}{\partial z_{1}^{2}}
$$


The classical boundary conditions $W^{(1)}=U_{2}^{(1)}=M_{11}^{(1)}=N_{11}^{(1)}=0$ at $z_{1}=0$ and $z_{1}=L$ are used in the present study. These boundary conditions are designated as $\mathrm{S} 2$ boundary conditions herein, following the notation given in Ref. 21. The symbols $U_{2}^{(1)}, M_{11}^{(1)}$, and $N_{11}^{(1)}$ represent the nondimensional circumferential displacement, the bending stress resultant, and the inplane stress resultant associated with the adjacent equilibrium states. Equations (5) and (6) are homogeneous partial differential equations with constant coefficients, and the boundary conditions are homogeneous partial differential operators with constant coefficients. Thus, equations (5) and (6) and the boundary conditions constitute a boundary-eigenvalue problem in which the loading parameter $\tilde{p}$ is the eigenvalue. Inspection of these equations, after expressing the boundary conditions in terms of the normal displacement and stress function, reveals that an exact solution is given by

$$
\begin{gathered}
F^{(1)}=B \sin \left(m \pi z_{1}\right) \sin \left(n z_{2}\right) \\
W^{(1)}=A \sin \left(m \pi z_{1}\right) \sin \left(n z_{2}\right)
\end{gathered}
$$

where $m$ is the integer number of axial half waves, $n$ is the integer number of circumferential full waves, and $A$ and $B$ are unknown modal amplitudes. Substituting Eqs. (7) and (8) into Eqs. (5) and (6) gives

$$
\begin{aligned}
& {\left[A C_{A}(m, n)+B C_{B}(m, n)\right] \sin \left(m \pi z_{1}\right) \sin \left(n z_{1}\right)=0} \\
& {\left[A C_{C}(m, n)+B C_{D}(m, n)\right] \sin \left(m \pi z_{1}\right) \sin \left(n z_{1}\right)=0}
\end{aligned}
$$

where $C_{A}(m, n), C_{B}(m, n), C_{C}(m, n)$, and $C_{D}(m, n)$ are coefficients that are functions of $\tilde{p}, m$ and $n$, the cylinder length and diameter, and the nondimensional parameters. Once values for $m$ and $n$ are chosen, the harmonic terms appearing in Eq. (9) are generally nonzero and, as a result, their coefficients must vanish. This requirement yields two homogeneous linear algebraic equations in $A$ and $B$. Nontrivial solutions may be found by setting the determinant of the coefficient matrix equal to zero and solving for $\tilde{p}$. The smallest positive value of the loading parameter represents the first intersection of an adjacent equilibrium path with the primary equilibrium path. In order to find the lowest bifurcation mode and load, $\tilde{p}_{b i f}$, a range of $m$ and $n$ are considered to find the $m$ and $n$ that give the lowest $\tilde{p}$.

\section{B. Nonlinear Deformations of Imperfect Cylinders}

The equations used herein to determine the nonlinear response of cylinders are also obtained by specializing the equations given in Ref. 20 for a generally anisotropic doubly curved shell to the case of a compression-loaded orthotropic cylinder. Following the same simplification procedure used to obtain Eqs. (1) and (2) gives

$$
\alpha_{b}^{2} \frac{\partial^{4} W}{\partial z_{1}^{4}}+2 \beta \frac{\partial^{4} W}{\partial z_{1}^{2} \partial z_{2}^{2}}+\frac{1}{\alpha_{b}^{2}} \frac{\partial^{4} W}{\partial z_{2}^{4}}+\sqrt{12} Z_{2} \frac{\partial^{2} F}{\partial z_{1}^{2}}=\frac{\partial^{2} F}{\partial z_{2}^{2}} \frac{\partial^{2}\left(W+W_{I}\right)}{\partial z_{1}^{2}}+\frac{\partial^{2} F}{\partial z_{1}^{2}} \frac{\partial^{2}\left(W+W_{I}\right)}{\partial z_{2}^{2}}-2 \frac{\partial^{2} F}{\partial z_{1} \partial z_{2}} \frac{\partial^{2}\left(W+W_{I}\right)}{\partial z_{1} \partial z_{2}}
$$

for the nonlinear nondimensional transverse equilibrium equation and

$$
\alpha^{2} \frac{\partial^{4} F}{\partial z_{1}^{4}}+2 \mu \frac{\partial^{4} F}{\partial z_{1}^{2} \partial z_{2}^{2}}+\frac{1}{a^{2}} \frac{\partial^{4} F}{\partial z_{2}^{4}}-\sqrt{12} Z_{2} \frac{\partial^{2} W}{\partial z_{1}^{2}}=\frac{1}{2}\left(\frac{\partial^{2} W}{\partial z_{2}^{2}} \frac{\partial^{2}\left(W+2 W_{I}\right)}{\partial z_{1}^{2}}+\frac{\partial^{2} W}{\partial z_{1}^{2}} \frac{\partial^{2}\left(W+2 W_{I}\right)}{\partial z_{2}^{2}}-2 \frac{\partial^{2} W}{\partial z_{1} \partial z_{2}} \frac{\partial^{2}\left(W+2 W_{I}\right)}{\partial z_{1} \partial z_{2}}\right)
$$

for the nonlinear nondimensional compatibility equation. In these two equations, $F$ is a nondimensional stress function defined in a manner analogous to Eq. (3), and $W_{I}$ and $W$ are the nondimensional radial imperfection and nondimensional radial displacement, respectively. Equations (10) and (11) are based on the approach used by Donnell ${ }^{14,15}$ in which relatively small initial shape imperfections are included through the use of an imperfection function defined as a radial displacement from the ideal cylinder middle surface. In this approach, the total radial displacement equals the sum of the radial imperfection function and the radial displacement produced by applied loads. The strain-displacement relations are formulated on the basis that the imperfection function cannot produce strains when applied loads are absent. Details of this approach are given in Ref. 20.

An important part of the approach used herein to solve Eqs. (8) and (9) is to represent the initial geometric imperfections $W_{I}$ in the form of the bifurcation buckling modes that are obtained by solving Eqs. (5) and (6); that is,

$$
W_{I}=\bar{f}_{I} \sin \left(m \pi z_{1}\right) \sin \left(n z_{2}\right)
$$

6

American Institute of Aeronautics and Astronautics 
where $\bar{f}_{I}$ is a specified nondimensional imperfection amplitude. This representation of the imperfections was chosen because the actual forms of the imperfections are unknown during the initial stages of the design process, and because the use of bifurcation modes is expected to yield conservative estimates of the imperfection sensitivity. Specifically, the bifurcation modes generally represent imperfections with a propensity for buckling that is greater than that associated with an actual imperfection (e.g., see Ref. 5).

Another important part of the approach used herein to solve Eqs. (10) and (11) is the rationale for selecting a relatively simple functional representation of the nonlinear radial displacement. For thin-walled closed cylinders buckled under axial load, the amplitude of the radially inward buckles is generally greater than the amplitude of the radially outward buckles. For this reason, $W$ must have a functional form with more deformational freedom than that given by Eq. (12) for the bifurcation mode. ${ }^{13,16}$ In this study, the functional form is the same as that used by Vol'mir; ${ }^{16}$ that is,

$$
W=f_{0}+f_{1} \sin \left(m \pi z_{1}\right) \sin \left(n z_{2}\right)+f_{2} \sin ^{2}\left(m \pi z_{1}\right) \sin ^{2}\left(n z_{2}\right)
$$

where $f_{0}, f_{1}$, and $f_{2}$ are unknown nondimensional radial-displacement amplitudes. The first term represents a uniform radial expansion, the second term is the same form as the bifurcation buckling displacement, and the third term is the additional term that allows the additional deformational freedom discussed above. It should be noted that this third term in Eq. (13) does not satisfy the condition that $M_{11}=0$ at $z_{1}=0$ and $z_{1}=L$. Vol'mir ${ }^{16}$ has stated that isotropic cylinders loaded in axial compression are generally insensitive to this condition.

Upon substituting Eqs. (12) and (13) into Eq. (11), the compatibility equation is converted into a nonhomogeneous partial differential equation with constant coefficients in terms of the stress function $F$. The method of undetermined coefficients is used to find a particular solution to the compatibility equation in terms of the unknown radial-displacement amplitudes $f_{0}, f_{1}$, and $f_{2}$. An approximate homogenous solution for $F$ is constructed by using the condition of periodicity of the circumferential displacement and by relaxing the boundary conditions on $N_{11}$ by allowing these boundary conditions to be satisfied in the average, integrated sense. This approach is based on the presumption that averaged representations of boundary conditions are sufficient for predicting global response quantities like buckling loads, and was adopted because of a substantial reduction in the mathematical complexity. Like that given by Vol'mir, ${ }^{16}$ the homogeneous solution, $F_{h}$, is approximated by

$$
F_{h}=-\frac{s}{2} z_{2}^{2}-\frac{t}{2} z_{1}^{2}
$$

where $s$ and $t$ are unknown coefficients that are related to the stresses in the axial and circumferential directions, respectively. If $s$ is chosen such that

$$
s=\pi^{2} \tilde{p}
$$

the boundary condition that $N_{11}=N_{11}^{\text {applied }}$ at $z_{1}=0$ and $z_{1}=L$ is satisfied in an averaged, integrated manner. This result is verified by substituting Eqs. (14) and (15) and the particular solution into the expression for $N_{11}$ in terms of the stress function and then integrating with respect to $z_{2}$ from 0 to $2 \pi$. The coefficient $t$ is determined in terms of $f_{0}$, $f_{1}$, and $f_{2}$ by enforcing periodicity of the circumferential displacement, as follows. First, the strain-displacement relations and constitutive equations are used to express the derivative of the circumferential displacement in terms of the $W$ and $F$. In particular, specializing the appropriate equations given in Ref. 20 to the cylinders considered herein yields

$$
\frac{\partial U_{2}}{\partial z_{2}}=-v_{m} \frac{\partial^{2} F}{\partial z_{2}^{2}}+\alpha_{m}^{2} \frac{\partial^{2} F}{\partial z_{1}^{2}}-\sqrt{12} Z_{2} W-\frac{1}{2}\left(\frac{\partial W}{\partial z_{2}}\right)^{2}-\frac{\partial W}{\partial z_{2}} \frac{\partial W_{1}}{\partial z_{2}}
$$

where $v_{m}$ is an additional nondimensional parameter given by

$$
v_{m}=\frac{A_{12}}{\sqrt{A_{11} A_{22}}}
$$

that is a generalized form of Poisson's ratio for overall in-plane deformations. The circumferential displacement is found by integrating Eq. (16) with respect to the $\mathrm{z}_{2}$ coordinate. After enforcing periodicity, it was verified that the 
stress function, given by Eq. (14) plus the particular solution, and the assumed displacement given by Eq. (13) satisfy the boundary condition $U_{2}=0$ at $z_{1}=0$ and $z_{1}=L$.

At this point in the analysis, the stress function $F$ and the radial displacement $W$ are known in terms of the unknown displacement amplitudes $f_{0}, f_{1}$, and $f_{2}$. The expressions for $W, W_{b}$ and $F$ are then substituted into the equilibrium equation, Eq. (10), and Galerkin's Method (see for example Ref. 22) is used to convert the nonlinear partial differential equation to three coupled nonlinear algebraic equations in terms of the unknowns $f_{0}, f_{1}$, and $f_{2}$. The first equation, using unity as the basis function, allows $f_{0}$ to be found in terms of $f_{1}$ and $f_{2}$. When this expression for $f_{0}$ is put into the expression for $t$, it is found that $t$ is identically zero, which indicates that there is no circumferential membrane stress in the cylinder. The coefficients of the unknowns in the remaining two equations depend on the values for the nondimensional parameters, the wave numbers $m$ and $n$, the imperfection amplitude $\bar{f}_{I}$, and the loading parameter $\tilde{p}$. Upon specifying these quantities, the two nonlinear algebraic equations can be solved. The real-valued roots of these equations define the corresponding nonlinear equilibrium configurations of a cylinder. The limit-point load is defined as the first local maximum on the primary equilibrium path of the imperfect cylinder that relates the applied load to the average axial end shortening. To obtain the nondimensional axial displacement $U_{1}$, the strain-displacement relations and constitutive equations are used to express the derivative of $U_{1}$ in terms of the $W$ and $F$. Specifically, specializing the appropriate equations given in Ref. 20 to the cylinders considered herein yields

$$
\frac{\partial U_{1}}{\partial z_{1}}=\frac{1}{\alpha_{m}^{2}} \frac{\partial^{2} F}{\partial z_{2}^{2}}-v_{m} \frac{\partial^{2} F}{\partial z_{1}^{2}}+\alpha_{m}^{2} \frac{\partial^{2} F}{\partial z_{1}^{2}}-\frac{1}{2}\left(\frac{\partial W}{\partial z_{1}}\right)^{2}-\frac{\partial W}{\partial z_{2}} \frac{\partial W_{I}}{\partial z_{2}}
$$

The average end shortening, $\delta$, is calculated by integrating Eq. (18) with respect to $z_{1}$ to get $U_{1}$ as a function of $z_{2}$. The resulting expression is then integrated around the circumference of the cylinder to get the average end shortening.

To further investigate the significance of violating the boundary condition $M_{11}=0$ at $z_{1}=0$ and $z_{1}=L$, the corresponding residual moment at each end of the cylinder was obtained. Then, the residuals were integrated around the circumference and found to be zero-valued.

\section{Baseline Results for an Isotropic Monocoque Cylinder}

As a first step in assessing the adequacy of the analysis presented herein, results were obtained for a monocoque aluminum cylinder with a radius $R=198$ in., a length $L=585 \mathrm{in}$., a thickness $h=0.6$ in., a Young's modulus $E=$ $10.4 \times 10^{6} \mathrm{psi}$, and a Poisson's ratio $v=0.3$. These dimensions are representative of an interstage for a heavy lift rocket that is capable of supporting an axial line load of $4500 \mathrm{lb}$./in. with a buckling knockdown factor of 0.65 and a factor of safety of 1.4. First, a detailed set of results obtained from the analytical model are presented to illustrate the solution methodology. For this effort, the analytical model was developed and solved by using the symbolic mathematical computer code Mathematica. ${ }^{23}$ Second, selected corresponding results obtained from finite-element analyses are presented and compared.

\section{A. Analysis Results}

The first step in assessing the analytical model is to compute the loads, and the associated modes, that correspond to bifurcation buckling of the idealized, geometrically perfect cylinder. The lowest bifurcation-buckling load and mode was found by considering all wave numbers for $1 \leq m \leq 20$ and $2 \leq n \leq 20$ that appear in Eqs. (7) and (8). For this highly axisymmetric cylinder, the lowest bifurcation-buckling load is given by $P_{b i f}=1.42 \times 10^{7} \mathrm{lbs}$. and the associated mode corresponds to the wave numbers $(m, n)=(9,15)$. In addition, the analysis predicted 79 modes with buckling loads within $5 \%$ of $P_{b i f}$ and 35 modes with buckling loads within $1 \%$ of $P_{b i f}$ for the ranges of wave numbers considered.

Next, nonlinear analyses of the idealized, geometrically perfect cylinder and the corresponding cylinder with a bifurcation-mode imperfection were conducted. In these analyses, a particular pair of wave numbers is specified, which yields a family of solution paths in the space spanned by the unknown displacement amplitudes, $f_{1}$ and $f_{2}$, and the load $P$. Each path in the family is determined by the value of the dimensional imperfection amplitude, $f_{I}$. Points of a solution path are obtained by specifying the load and by then solving the nonlinear algebraic equations that result from the application of Galerkin's Method for $f_{1}$ and $f_{2}$. For a given load value, there are nine roots to these equations, for which the real-valued roots define the possible equilibrium configurations of the cylinder. A complete solution path is obtained by solving these nonlinear equations repeatedly for monotonically increasing values of the load over the desired loading range. 
Two solution paths of the family defined by $(m, n)=(9,15)$ are shown in Figs. $3 \mathrm{a}$ and $3 \mathrm{~b}$ for a perfect cylinder with $f_{I}=0$ and an imperfect cylinder with $f_{I}=0.05 h$, respectively, where the load is normalized by $P_{b i f}$. In addition, the normalized-load vs. end-shortening curves are shown in Figs. $3 \mathrm{f}$ and $3 \mathrm{~g}$, the normalized load vs. $f_{1}$ is shown in Figs. $3 \mathrm{c}$ and $3 \mathrm{~d}$, and the normalized load vs. $f_{2}$ in shown in Figs. 3e and 3f. The curves shown in Figs. $3 \mathrm{c}$ through $\mathrm{f}$ represent projections of the solution path onto a plane, and as such the curves cannot be traversed in a manner where either $f_{1}$ or $f_{2}$ is held at a fixed value. Collectively, the plots for the perfect cylinder are shown on the left side of Fig. 3 and for the imperfect cylinder on the right. The primary equilibrium paths are shown in blue and the secondary paths are shown in red, and the curves are represented by individual points for the each of the real solutions obtained for a given value of $\tilde{p}$. Because the paths are represented by individual points, some of the paths may appear discontinuous in areas of near-zero slope; if a smaller interval was chosen for $\tilde{p}$, these apparent discontinuities would diminish.

The results in Fig. 3 for the geometrically perfect cylinder under increasing load indicate that the solution path consists of three connected branches. In particular, the solution path follows the line from point A, which corresponds to no loading, to the bifurcation point at point B shown in the figures. Once point B is reached, two new paths $\mathrm{BC}$ and $\mathrm{BD}$ exist that have identical load-response characteristics; this is consistent with an unstable symmetric bifurcation. Specifically, paths BC and BD represent the same response of the cylinder, except that the values of $f_{1}$ for path $\mathrm{BC}$ are the negatives of the corresponding values for path $\mathrm{BD}$. The effect of this similarity yields deformed shapes that are identical but offset by a rotation of one circumferential half wavelength, i.e., offset by $\theta=\pi / n$.

The response of the imperfect cylinder to loading shows some similarities with that of the perfect cylinder, however there are important differences. For example, the imperfect solution path consists of two disconnected branches. When an imperfection is present, bending and membrane deformation occur at the onset of loading. As such, it is seen that the primary equilibrium path $\mathrm{AB}$ corresponds to nonzero values of $f_{1}$ and $f_{2}$. Additionally, the results indicate that point $B$ is a limit point of the imperfect-cylinder response. At this point, further traversal of the solution path cannot sustain an increase in the load and the cylinder must go into motion to acquire a stable equilibrium configuration. Thus, buckling of the imperfect cylinder occurs at the limit point, and the corresponding buckling load is denoted by $P_{l i m}$. It is important to note that $P_{l i m}$ is significantly smaller than $P_{b i f}$. Thus, the ratio $P_{l i m} / P_{b i f}$ represents the sensitivity of the cylinder to the initial geometric imperfection and is referred to herein as the imperfection sensitivity factor. It should also be noted that the only effect of reversing the sign of the initial imperfection amplitude is to interchange the position of the red and blue curves in Fig. 3b. The corresponding mode shape is offset by one circumferential half wavelength.

The effects of varying the magnitude of the bifurcation-mode-imperfection amplitude on the load-response curves and the imperfection sensitivity are shown in Fig. 4. Five load-response curves that correspond to values of imperfection amplitudes bounded by $f_{I}=0$ and $0.20 h$ are shown in Fig. 4a. Each of these load-response curves corresponds to the bifurcation mode given by $(m, n)=(9,15)$. In this figure, point A corresponds to the bifurcation buckling load and points $B$ through $E$ correspond to the limit points of the equilibrium paths defined by imperfection amplitudes between $f_{I}=0.01 h$ and $0.11 h$. The results indicate that the load-response curves for the very small imperfection amplitudes extend far into the cusp, toward $P_{b i f}$, of the corresponding curve for the perfect cylinder. However, as the imperfection amplitude increases, the load-response curves move away from the perfect-cylinder curve, the post-limit-load drop in load diminishes, and the imperfection sensitivity increases. For $f_{I}=0.11 h$, the bend in the load-response curve is almost completely flat. For imperfection amplitudes larger than $0.11 \mathrm{~h}$, there is no limitpoint behavior. For example, for $f_{I}=0.20 \mathrm{~h}$ there is an inflection point at point $\mathrm{F}$ of the load-response curve, but no limit point. This equilibrium path is characteristic of a stable monotonically increasing response.

These effects of imperfection amplitude on the imperfection sensitivity, or reduction in buckling resistance, for mode $(m, n)=(9,15)$ are shown as the imperfection sensitivity factor plotted vs. imperfection amplitude in Fig. $4 \mathrm{~b}$. The colored, lettered points on this curve correspond to the same colored and lettered points on Fig. 4a. It is seen that for increasing initial imperfection amplitudes there is a monotonic reduction in the ordinate until point $\mathrm{E}$ is reached, which corresponds to a monotonic increase in the imperfection sensitivity. For larger imperfection amplitudes, there is no predicted limit point for mode $(9,15)$. Overall, the maximum imperfection sensitivity shown in Fig. $4 \mathrm{~b}$ corresponds to a reduction in load-carrying capacity of approximately $24 \%$ for an imperfection amplitude that is approximately a tenth of the wall thickness.

Observing the load-response curves obtained for different modal imperfections indicates that the imperfection sensitivity associated with a of range of modes must examined in order to get a conservative estimate of the overall imperfection sensitivity. Toward this goal, consider the load-response curves for a geometrically perfect cylinder obtained for the wave numbers $(9,15),(9,13),(9,10),(7,9)$ that are shown in Fig. 5. These curves represent modes that produce a range of imperfection sensitivities that is broader than that obtained by considering the bifurcation 
mode given by $(9,15)$. The black postbuckling branch in the figure corresponds to $(m, n)=(9,15)$, associated with $P_{b i f}$. Thus, the black postbuckling curve intersects the straight prebuckling line at $P=P_{b i f}$. In contrast, the bifurcation load of the $(9,13)$ mode is about $8 \%$ higher than $P_{b i f}$, but the postbuckling curve dips well below that of mode $(9$, 15). Similarly, the bifurcation loads for modes $(9,10)$ and $(7,9)$ modes are considerably higher than $P_{b i f}$, and their postbuckling branches also dip below that of the $(9,15)$ mode. As will be seen in Fig. 6 , each of these modes produces the lowest limit-point load for some value of imperfection amplitude. In particular, in order of increasing imperfection amplitude, modes $(9,15),(9,13),(9,10)$, and $(7,9)$ produce the lowest limit-point load.

Though certainly not all inclusive, the four postbuckling curves shown in Fig. 5 strongly suggest that a mode other than the bifurcation mode may yield a greater imperfection sensitivity than the bifurcation mode, and that the wave numbers associated with the smallest limit-point load are likely to change as the size of the initial imperfection amplitude changes. Therefore, if bifurcation-mode imperfections are used to estimate imperfection sensitivity, the extreme sensitivity must be determined by examining the limit-point loads associated with all relatively nearby modes over a given range of wave numbers and imperfection amplitudes. This idea is somewhat justified by noting that experiments using high-speed photography to capture the progression of the postbuckling of compressionloaded cylinders have shown that the initial buckle pattern was observed to consist of small, high-wave-number buckles similar to the predicted bifurcation mode. However, as the buckling progressed, the deformation pattern transitioned rapidly through larger, lower-wave-number buckles until the stable post buckling configuration was reached (see Ref. 24).

To obtain the extreme imperfection sensitivity, the smallest limit-point load corresponding to a value of the imperfection amplitude $f_{I}$ were determined for all modes given by $1 \leq m \leq 20$ and $2 \leq n \leq 20$. The resulting imperfection sensitivity factors, $P_{l i m} / P_{b i f}$, are plotted in Fig. 6 for imperfection amplitudes between zero and one wall thickness, and selected results are shown in Table 1. The lower-bound envelope of the results is shown as the thick black line connecting diamond-shaped symbols. For each point of the envelope, the values of $(m, n)$ are shown in the figure. The grey dashed lines shown, extending up and to the left from each points of the envelope, represent $P_{\text {lim }} / P_{b i f}$ for that particular mode. Moreover, each of these grey dashed lines is drawn over the range of imperfection amplitudes in which the response exhibits a limit point. The blue curve corresponds to the results presented in Fig. 5.

The results in Fig. 6 indicate that the minimum of the ratio $P_{l i m} / P_{b i f}$ denoted by $P_{l i m}^{\min } / P_{b i f}$ approaches unity as the imperfection amplitude approaches zero. As the imperfection amplitude increases, there is a significant, and generally monotonic, reduction in $P_{l i m}^{\min } / P_{b i f}$. In particular, as the imperfection amplitude approaches one wall thickness, $P_{l i m} / P_{b i f}$ approaches 0.4 . This value of 0.4 corresponds to a $60 \%$ reduction in load-carrying capacity, and is very close to the knockdown factor given in NASA SP-8007 (knockdown factor $=0.39$ ) indicated by the green line in the figure. However, the results also show that there are several slight increases $P_{l i m}^{\min } / P_{b i f}$. It is seen that these increases in $P_{\text {lim }}^{\min } / P_{b i f}$ occur at imperfection amplitudes where the limit-point behavior of the mode that gives $P_{\text {lim }}^{\min }$ terminates. Additionally, at the imperfection amplitudes where these increases occur, the imperfection-amplitude range over which the many of the modes show limit-point behavior has been exceeded. This observation suggests the need to have clustered eigenvalues and many available mode-shape paths if the current methodology is to show a smooth monotonic imperfection-sensitivity response to changes in imperfection amplitude. That is, if few modes produce limit-point behavior at a particular imperfection amplitude, these increases in $P_{\text {lim }}^{\min } / P_{b i f}$ may be large.

\section{B. Finite-Element Analysis}

In order to check the analytical model and to begin to determine its range of validity, the predicted imperfection sensitivities obtained by using the analytical model were compared with corresponding results from finite-element analyses. The general-purpose finite-element code STAGS version $5.0^{25}$ was used for this comparison. As with the analytical model, two types of analyses were run with STAGS - linear eigenvalue analyses to find the bifurcation buckling load, and geometrically nonlinear analyses to find the limit-point load for imperfect cylinders. For all analyses the STAGS E410 four-node quadrilateral shell element with no transverse-shear flexibility was used. The mesh consisted of 29,500 approximately square elements -118 elements in the axial direction and 250 elements in the circumferential direction. Additionally, the S2 simply supported boundary conditions were used for both types of finite-element analysis. To ensure that the STAGS results are accurate, a convergence study was conducted. As summarized in Table 2, significantly coarser and finer meshes were considered for both the linear eigenvalue analysis and the nonlinear analysis with an imperfection in the form of Eq. $(12)$ with $(m, n)=(1,6)$ and an imperfection amplitude of $f_{I}=0.33 \mathrm{~h}$. The calculated bifurcation buckling modes and loads and nonlinear limit-point loads were each found to vary less than $2 \%$ for the three different meshes.

For the nonlinear STAGS analysis of the imperfect cylinder, two user subroutines are used. The first user subroutine applies an initial imperfection in the form of Eq. (12). The second user subroutine prevents solutions with 
negative eigenvalues from being accepted, so that the solver attempts to find stable solutions as long as they exist. Past institutional experience has shown that this subroutine in conjunction with the STAGS solver will usually follow a stable path to very near the limit-point load. Thus, the last converged solution was taken to be the limitpoint load in this study. In order to find the imperfection-mode wave numbers that gives the minimum limit-point load, a different approach from that of the analytical model had to be taken. That is, because of the analysis time required, it was infeasible to analyze all modes for $1 \leq m \leq 20$ and $2 \leq n \leq 20$, so a subset of these modes was chosen. Specifically, the minimum limit-point load was found in the vicinity of the modes that lead to each of the analytical-model $P_{b i j}$, analytical-model $P_{l i m}^{\min }$, and the STAGS $P_{b i f}$. Each of these three starting modes and all the immediately neighboring modes in $m$ and $n$ were used as the initial imperfection shape in separate STAGS analyses. For example, in the analytical model, the mode $(m, n)=(9,15)$ gave $P_{b i f}$. To examine the imperfection sensitivity for other modes near this one, separate nonlinear STAGS analyses were run to find the limit-point loads for nine different imperfection modes in the vicinity of $(9,15)$, i.e., in the mode space bounded by $8 \leq m \leq 10$ and $14 \leq n \leq$ 16. Likewise, nonlinear STAGS analyses were run for each of the nine initial-imperfection modes in the immediate vicinity of the $(m, n)$ that led to both the analytical-model $P_{\text {lim }}^{\min }$ and the STAGS $P_{b i f}$. If the mode that led to the STAGS lowest limit-point load was on the edge of one of these mode spaces, that row in $m$ or $n$ was expanded out until a local minimum in the limit-point load was found. Though this method did not span the entire space that the analytical model did, it appears to be a reasonable method of finding the STAGS $P_{\text {lim }}^{\min }$ because the surface created by plotting the limit-loads vs. $m$ and $n$ is generally smooth, and fairly flat near this minimum limit-point load.

The STAGS linear eigenvalue analysis predicted the bifurcation buckling load of the aluminum cylinder to be $P_{b i f}=1.36 \times 10^{7} \mathrm{lbs}$., which is approximately $4 \%$ lower than the bifurcation buckling load predicted by the analytical model. This difference is attributed to approximations made in the Donnell equations and the use of a membrane prebuckling state in the analytical model, which neglects bending at the ends of the cylinder in the prebuckling solution. Nonlinear analyses of the imperfect shells were conducted for the three initial imperfection amplitudes $f_{I}=$ $0.165 h, 0.33 h$, and $0.66 h$. These imperfection amplitudes are equivalent to initial imperfection amplitudes of $R / 2000$, $R / 1000$, and $R / 500$, and were selected to facilitate comparisons with results for composite shells that were obtained in the present study and discussed in the next section. For these three initial imperfection amplitudes, the smallest limit-point loads $P_{\text {lim }}^{\min }$ obtained were $7.67 \times 10^{6} \mathrm{lbs}$., $5.80 \times 10^{6} \mathrm{lbs}$., and $4.08 \times 10^{6} \mathrm{lbs}$., respectively. The corresponding imperfection sensitivity factors are $0.57,0.43$, and 0.30 , and are also plotted in Fig. 6 . These results show that the imperfection sensitivity factors predicted by STAGS are lower than those predicted by the analytical model. However, both analysis methods predict a similar trend. In addition, the differences increase as the imperfection amplitude increases. These differences are likely caused by the greater deformational freedom and the prebuckling bending deformations at the cylinder ends that are included in the STAGS analyses. The results in Fig. 6 also show that the knockdown factor recommended by NASA SP-8007 is close to lowest value of imperfection sensitivity factor predicted by the analytical model, but slightly higher than that predicted by STAGS.

Additional finite-element analyses were conducted in the present study to examine the significance of satisfying the intended boundary conditions only in an average manner in the analytical model. In particular, this concern was addressed by examining the limit-point loads obtained for the end conditions of $M_{11}=0$ and $\partial W / \partial z_{1}=0$. This change in the boundary conditions led to less than $1 \%$ difference in the limit-point loads for modes $(m, n)=(1,6)$ and $(13,15)$ with $f_{I}=0.33 h$.

The finite-element analyses also demonstrated the need for a special-purpose analysis tool that can be used in a design setting. For example, on a LINUX-based computer, the STAGS coarse-mesh model took more than 5 minutes to run, the medium-mesh model took between 40 and 60 minutes, and the fine-mesh model took over 3 hours for each imperfection mode considered. These run times do not include any postprocessing or other interaction time needed to run the analyses and get usable output. On the same computer, the analytical model takes between 40 and 60 minutes to run analyses for all imperfection modes over $1 \leq m \leq 20$ and $2 \leq n \leq 20$.

\section{Imperfection Sensitivity Estimates for Composite Sandwich Cylinders}

To demonstrate the utility of the analytical model presented herein, while also assessing its validity, results for three sandwich composite cylinders that are potential candidates for new launch-vehicle components are presented in this section. The face sheets consist of unidirectional graphite-epoxy plies and the core is $3.1 \mathrm{pcf}$ aluminum honeycomb. The structural optimization code PANDA2 ${ }^{26}$ was used to size the face-sheet and core thicknesses to support an axial line load of $4500 \mathrm{lb}$./in. and to minimize the structural mass. A buckling knockdown factor equal to 0.65 and a factor of safety equal to 1.4 were also used. The optimization study considered three wall constructions with symmetric face sheets. The face-sheet layups for the three sandwich cylinders are a $[ \pm 45 / 0 / 90]_{2 \mathrm{~s}}$ quasi-isotropic layup with $25 \%$ axial plies, a $[ \pm 45 / 0 / 90 / 0 / 90 / 0]_{s}$ layup with $43 \%$ axial plies, referred to herein as the tailored 
cylinder, and a $[45 / 0 /-45 / 0 / 0 / 90 / 0 / 0 / 90 / 0]_{\mathrm{s}}$ layup with $60 \%$ axial plies, referred to herein as the highly tailored cylinder. The core thicknesses and total thicknesses for the three shells are given in Table 1. Relative to the quasiisotropic cylinder the tailored cylinder has a $10 \%$ lower mass, and the highly tailored cylinder has a $7 \%$ lower mass. The three sandwich shells considered have, comparatively, very small $A_{16}, A_{26}, D_{16}, D_{26}$, and extension-bending coupling ( $B$-matrix) stiffness terms. As a result, these stiffnesses are neglected in the analytical model and the finiteelement analyses.

The procedure used to determine the imperfection sensitivity of the composite sandwich cylinders was identical to that presented previously for the isotropic cylinder, and as presented in Table 3, a finite-element convergence study was also conducted for the quasi-isotropic shell by considering the same three meshes used with the isotropic cylinder. In this convergence study, a linear eigenvalue analysis and the nonlinear analysis with an imperfection in the form of Eq. (12) with $(m, n)=(6,7)$ and an imperfection amplitude of $f_{I}=0.11 h$ were performed for each mesh. The calculated bifurcation modes and buckling loads and nonlinear limit-point loads were each found to vary by about $1 \%$ or less for the three different meshes.

The bifurcation buckling loads predicted with the analytical model, for all modes numbers over $1 \leq m \leq 20$ and 2 $\leq n \leq 20$, and with the STAGS finite-element code for the three composite sandwich cylinders are presented in Table 1. These results indicate differences in the predictions obtained by the two analysis methods of $13 \%, 3.8 \%$, and 4.7\% for the quasi-isotropic, tailored, and highly tailored sandwich cylinders, respectively. Like for the isotropic cylinder, these differences are attributed to approximations made in the Donnell equations and the use of a membrane prebuckling state in the analytical model, which neglects bending at the ends of the cylinder in the prebuckling solution.

Next, the lowest imperfection sensitivity factor, which corresponds to the smallest limit-point load $P_{\text {lim }}^{\min }$, was calculated over a range of initial imperfection amplitudes by using the analytical model with bifurcation modes defined by $1 \leq m \leq 20$ and $2 \leq n \leq 20$; STAGS was used to correlate the results for three initial imperfection amplitudes. Additionally, buckling knockdown factors were calculated by using the equations given in NASA SP8007 for an isotropic sandwich structure. These values are equal to $0.64,0.69$, and 0.72 for the quasi-isotropic, tailored, and highly tailored cylinders, respectively, and are shown in Figs. 7a, $7 \mathrm{~b}$, and $7 \mathrm{c}$, respectively, as the horizontal green lines. The black lines in Fig. 7 represent the envelope of imperfection sensitivity factors obtained from the analytical model, and the red circles represent the corresponding results obtained with STAGS. The imperfection amplitude range shown in Fig. 7 is for $f_{I}$ between zero and $0.25 h$. This range was chosen because the three composite sandwich shells are all relatively thick and because the bifurcation modes that yield the smallest limit-point loads are high-frequency modes. As a result, a high-frequency imperfection shape with an amplitude equal to $25 \%$ of the wall thickness is approaching the limit of acceptable manufacturing tolerances for this class of cylinders. Additionally, the Fourier cosine series representations of measured cylinder imperfections given in Ref. 5 indicate that the dominant waveform comprising the measured data (other than the elliptical waveform not considered herein) corresponds to the $(1,2)$ bifurcation mode with an amplitude equal to $20 \%$ of the wall thickness. Moreover, the measured data in Ref. 5 indicates that as the wave number increases in either the axial or circumferential direction, the amplitude quickly diminishes.

The results in Fig. 7 and Table 1 indicate that the analytical and finite-element predictions are in fairly good agreement for all three sandwich cylinders and exhibit the same trends. Specifically, the imperfection sensitivity factors predicted by STAGS were lower than the corresponding ones predicted by the analytical model, for all three sandwich constructions and the full range of imperfection amplitudes. The agreement was within $9 \%$ for the tailored and highly tailored sandwiches, and within $25 \%$ for the quasi-isotropic sandwich. For all three sandwich cylinders, the agreement is within $10 \%$ for initial imperfection amplitudes of $R / 2000$. The results also show that the quasiisotropic sandwich is predicted to be the most imperfection sensitive and the highly tailored sandwich is predicted to be the least imperfection sensitive. However, the significance of this difference is somewhat obscured because the imperfection amplitudes in Fig. 7 are normalized by the wall thickness that is different for the three sandwiches. For example, consider the results presented in Fig. 8. These results correspond to the imperfection amplitudes used to obtain the circular red symbols shown in Fig. 7. Specifically, imperfection sensitivity factors are given in Fig. 8 for the isotropic and the three sandwich cylinders, and are shown for imperfection amplitudes of $R / 2000, R / 1000$, and $R / 500$. Representing the imperfection amplitude in this way allows direct comparison in terms of physically observable imperfection amplitudes. With this representation as a basis for comparison, the results in Fig. 8 show in a clear manner that the isotropic cylinder is predicted to be the most imperfection sensitive and the highly tailored cylinder is predicted to be the least imperfection sensitive. Additionally, the results in Fig. 8 show that there is generally better agreement between the analytical and finite-element predictions as the amount of tailoring increases, that is, moving left to right in Fig. 8. 


\section{Design Implications}

The results in Fig. 7 also indicate that the buckling knockdown factors recommended by NASA SP-8007 are lower than those predicted by the analytical model, over the entire range of imperfection amplitudes considered, for all three sandwich constructions. However, for imperfection amplitudes of $025 h$, the imperfection sensitivity factors for the three sandwich cylinders were within $4 \%$ of the buckling knockdown factors obtained from NASA SP-8007. It should be noted that the NASA SP-8007 recommendations are shown for reference, and that the imperfection sensitivity factors given in this paper are not intended to be used as replacements. However, the results do indicate the potential advantages of developing refined imperfection sensitivity estimates for use in design. For example, the three composite sandwich cylinders considered herein were designed using a buckling knockdown factor of 0.65. Based on this knockdown factor, the tailored cylinder is predicted to yield the minimum mass. In contrast, the results in Figs. 7 and 8 predict the highly tailored cylinder to be less imperfection sensitive, suggesting that a higher knockdown factor could be used to design the highly tailored cylinder. To demonstrate the potential benefits of using refined imperfection sensitivity knockdown factors on the shell design, PANDA2 was used to find optimal designs for the three sandwich layups over buckling knockdown factors ranging from 0.6 to 0.9 . This range of knockdown factors is representative of the predictions given in Fig. 7. The results of the PANDA2 analyses are shown in Fig. 9 as the areal weight (weight per unit area) plotted as a function of the buckling knockdown factor for the three sandwiches. With respect to a knockdown factor of 0.65 , these results show $7.1 \%, 11 \%$, and $16 \%$ weight savings for the quasi-isotropic, tailored, and highly tailored cylinders, respectively, as the knockdown factor approaches 0.9. In addition, the highly tailored sandwich family might lead to lower mass than the tailored sandwich. That is, by being able to move farther to the right in Fig. 9, the tailored shell construction might lead to a lower mass. Regardless of whether the tailored or highly tailored laminate families would be better, comparing either one with the quasi-isotropic cylinder shows that some tailoring may lead to a "double benefit" of mass reduction due to both the tailoring itself and to the reduced imperfection sensitivity. These are the type of potential benefits that can be explored with a special-purpose tool that captures the essence of the nonlinear buckling behavior of cylinders.

\section{Concluding Remarks}

It has long been known that geometric imperfections can cause the load-carrying capabilities of inherently imperfect real structures to be much lower than theoretical predictions for perfect structures. For the design of shell structures, buckling knockdown factors that were developed in the 1960's are used to account for this imperfection sensitivity, and there are efforts at NASA and elsewhere to develop analysis-based knockdown factors based on characteristic geometric imperfections. In the present paper, the development of an analytical model for assessing the imperfection sensitivity of axially compressed orthotropic cylinders and results from the model have been discussed. The analytical model uses bifurcation buckling modes as initial geometric imperfections and a relatively simple form for the assumed displacements. Results for an isotropic cylinder and several composite sandwich cylinder designs with varying degrees of stiffness tailoring have been presented. These results were compared with results from the general-purpose finite-element code STAGS, and reasonable agreement and the same trends are seen with both analysis techniques. However, the imperfection sensitivity is consistently under predicted with the analytical model. In this paper, the results have been presented in terms of an imperfection sensitivity factor (the imperfect limit load divided by the bifurcation buckling load). This factor is not intended to be used in place of the buckling knockdown factors of NASA SP- $8007^{1}$, but rather is intended to be a relative measure the imperfection sensitivity of different shells.

The four cylinders considered in this study had the same shell dimensions and were designed to meet realistic heavy-lift launch vehicle loads. The three composite sandwich cylinders had face sheets with different degrees of axial stiffness and were sized to minimize mass for that layup. For the cylinders examined in this study, the isotropic cylinder showed the highest degree of imperfection sensitivity. For the composite cylinders, the quasi-isotropic sandwich was predicted to have the highest imperfection sensitivity and the most axially stiff cylinder was predicted to have the lowest imperfection sensitivity. This result indicates that it should be possible to design composite cylinders that benefit from tailoring both to meet the design loads and also to reduce the imperfection sensitivity.

\section{Acknowledgments}

The authors would like to thank Waddy T. Haynie of the NASA Langley Research Center for his help with the STAGS analyses. Additionally, Mark Hilburger, also of NASA Langley, is to be thanked for helpful suggestions, comments, and conversations regarding this work. Finally, it should be mentioned that Prasad B. Chunchu of Eagle Aeronautics conducted the PANDA2 optimizations. 


\section{References}

${ }^{1}$ Anon. "Buckling of Thin-Walled Circular Cylinders. NASA Space Vehicle Design Criteria," NASA SP-8007, 1965, revised 1968.

${ }^{2}$ Anon. "Buckling of Thin-Walled Truncated Cones. NASA Space Vehicle Design Criteria," NASA SP-8019, 1968.

${ }^{3}$ Anon. "Buckling of Thin-Walled Doubly Curved Shells. NASA Space Vehicle Design Criteria/Structures." NASA SP-8032, 1969.

${ }^{4}$ Nemeth, M. P., and Starnes, J. H., "The NASA Monographs on Shell Stability Design Recommendations." NASA/TP-1998206290, January 1998.

${ }^{5}$ Hilburger, M. W., Starnes, J. H., and Nemeth, M. P., "Shell Buckling Design Criteria Based on Manufacturing Imperfection Signatures," NASA/TM-2004-212659, May 2004.

${ }^{6}$ Arbocz, J. and Hol, J. M. A. M., "On a Verified High-Fidelity Analysis for Axially Compressed Orthotropic Shells," Proceedings of the $46^{\text {th }}$ AIAA/ASME/ASCE/AHS/ASC Structures, Structural Dynamics \& Materials Conference, Austin, TX, April 2005, AIAA Paper No. 2005-2302.

${ }^{7}$ Cohen, G. A., "Computer Analysis of Imperfection Sensitivity of Ring-Stiffened Orthotropic Shells of Revolution," $A I A A$ Journal, Vol. 9, No. 6, 1971, pp. 1032-1039.

${ }^{8}$ Shulga, S. A., Sudol, D. E., Nishino, F., "Influence of the Mode of Initial Geometrical Imperfections on the Load-Carrying Capacity of Cylindrical Shells Made of Composite Materials," Thin-Walled Structures, Vol. 14, 1992, pp. 89-103.

${ }^{9}$ Biagi, M. and Del Medico, F., "Reliability-Based Knockdown Factors for Composite Cylindrical Shells Under Axial Compression," Thin-Walled Structures, Vol. 46, 2008, pp. 1351-1358.

${ }^{10}$ Huang, H. and Han, Q., "Buckling of imperfect functionally graded cylindrical shells under axial compression," European Journal of Mechanics A/Solids, Vol. 27, 2008, pp. 1026-1036.

${ }^{11}$ Shen, H.-S., "Boundary layer theory for the buckling and postbuckling of an anisotropic laminated cylindrical shell. Part I: Prediction under axial compression," Composite Structures, Vol. 82, 2008, pp. 346-361.

${ }^{12}$ Donnell, L. H., "A New Theory for the Buckling of Thin Cylinders Under Axial Compression and Bending," Transactions of the American Society of Mechanical Engineers, Vol. 56, 1934, pp. 795-806.

${ }^{13}$ von Kármán, T., and Tsien, H.-S., "The Buckling of Thin Cylindrical Shells Under Axial Compression," Journal of the Aeronautical Sciences, Vol. 8, No. 8, 1941, pp. 303-312.

${ }^{14}$ Donnell, L. H., and Wan, C. C., "Effect of Imperfections on Buckling of Thin Cylinders and Columns Under Axial Compression," Journal of Applied Mechanics, Vol. 17, 1950, pp. 73-83.

${ }^{15}$ Donnell, L. H., Beams, Plates, and Shells, McGraw-Hill, New York, 1976, Chaps. 6, 7.

${ }^{16}$ Vol'mir, A. S., Flexible Plates and Shells, translated by the Department of Engineering Science and Mechanics, University of Florida, Air Force Flight Dynamics Laboratory Technical Report, AFFDL-TR-66-216, 1967, Wright-Patterson Air Force Base, Ohio, Chaps. V, VI, VII, VIII.

${ }^{17}$ Singer, J., Arbocz, J., and Weller, T., Buckling Experiments: Experimental Methods in Buckling of Thin-Walled Structures Volume 1, Wiley, New York, 1998, Chap. 3.

${ }^{18}$ Timoshenko, S. P., and Gere, J. M., Theory of Elastic Stability, $2^{\text {nd }}$ ed., McGraw-Hill, New York, 1961, Chap. 11.

${ }^{19}$ Nemeth, M. P., "Nondimensional Parameters and Equations for Buckling of Symmetrically Laminated Thin Elastic Shallow Shells," NASA TM-104060, 1991.

${ }^{20}$ Nemeth, M. P., "Nondimensional Parameters and Equations for Nonlinear and Bifurcation Analyses of Thin Anisotropic Quasi-Shallow Shells," NASA TM, under review, to be published.

${ }^{21}$ Jones, R. M., Buckling of Bars, Plates, and Shells, Bull Ridge Publishing, Blacksburg, VA, 2006.

${ }^{22}$ Meirovich, L., Computational Methods in Structural Dynamics, Springer-Verlag, New York, 1980. Chap 8.

${ }^{23}$ Wolfram Mathematica, Software Package, Ver. 7.0, Wolfram Research, Champaign, IL, 2009.

${ }^{24}$ Singer, J., Arbocz, J., and Weller, T., Buckling Experiments: Experimental Methods in Buckling of Thin-Walled Structures Volume 2, John Wiley \& Sons, New York, 2002, Sect. 9.2.1, pp. 631-640.

${ }^{25}$ Rankin, C. C., Brogan, F. A., Loden, W. A., and Cabiness, H. D., "STAGS Users Manual, Version 5.0," Report LMSC P032594, Lockheed-Martin Missiles \& Space Co., March 1999.

${ }^{26}$ Bushnell, D., "PANDA2 - Program for Minimum Weight Design of Stiffened, Composite, Locally Buckled Panels," Computers and Structures, Vol. 25, No. 4, pp 469-605, 1987. 
Table 1: Shell designs, nondimensional parameters, and analysis results.

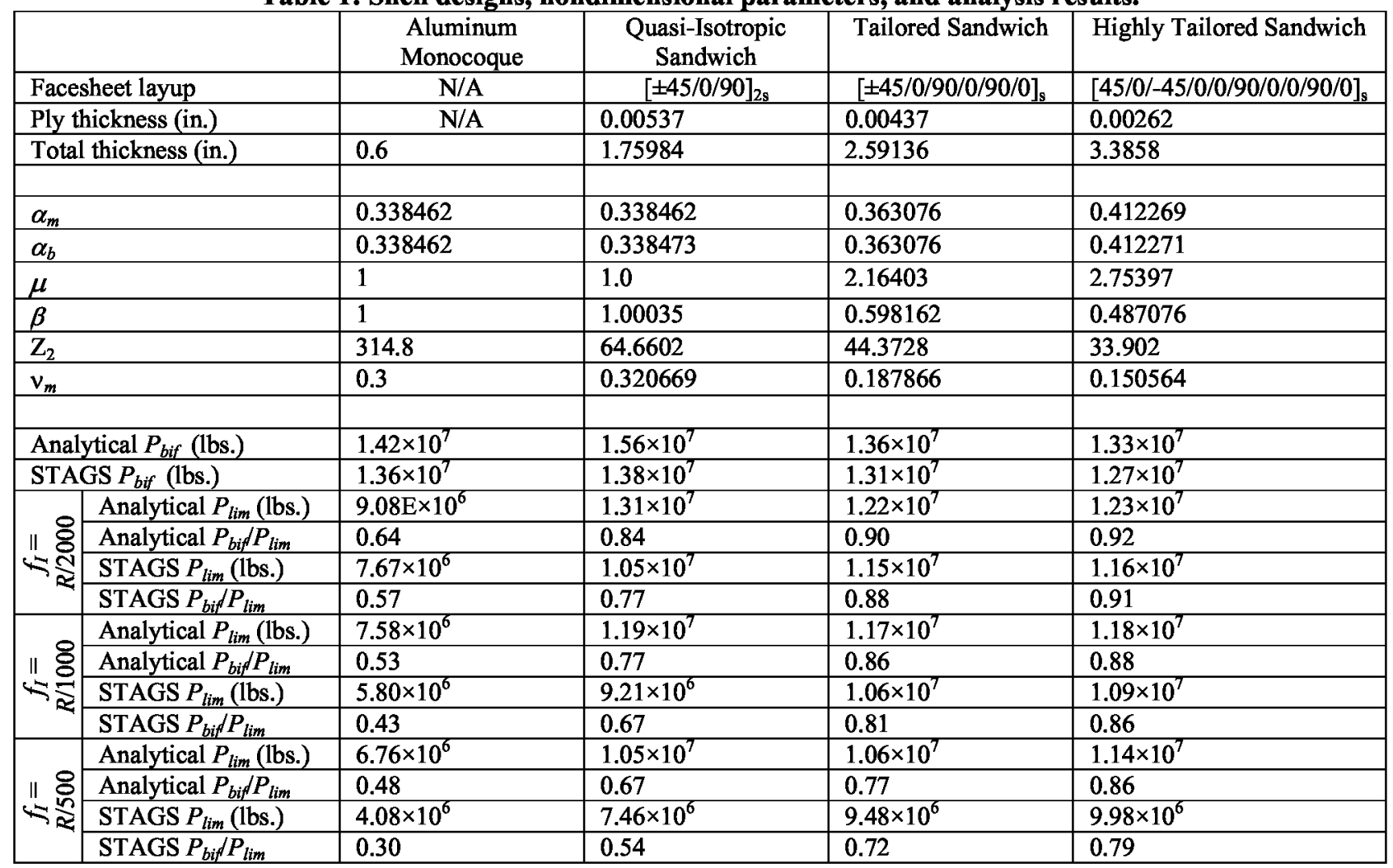

Table 2: STAGS mesh convergence study for the isotropic cylinder.

\begin{tabular}{|l|l|l|l|}
\hline & Coarse Mesh & Medium Mesh & Fine Mesh \\
\hline Axial elements & 60 & 118 & 196 \\
\hline Circumferencial elements & 125 & 250 & 416 \\
\hline Total elements & 7500 & 29500 & 81536 \\
\hline Bifurcation buckling load, lbs. & $1.37 \times 10^{7}$ & $1.36 \times 10^{7}$ & $1.35 \times 10^{7}$ \\
\hline Bifurcation buckling mode, $(m, n)$ & $(1,6)$ & $(1,6)$ & $(1,6)$ \\
\hline Limit-point load, lbs. & $1.10 \times 10^{7}$ & $1.09 \times 10^{7}$ & $1.09 \times 10^{7}$ \\
\hline
\end{tabular}

Table 3: STAGS mesh convergence study for the quasi-isotropic sandwich cylinder.

\begin{tabular}{|l|l|l|l|}
\hline & Coarse Mesh & Medium Mesh & Fine Mesh \\
\hline Axial elements & 60 & 118 & 196 \\
\hline Circumferencial elements & 125 & 250 & 416 \\
\hline Total elements & 7500 & 29500 & 81536 \\
\hline Bifurcation buckling load, lbs. & $1.38 \times 10^{7}$ & $1.37 \times 10^{7}$ & $1.37 \times 10^{7}$ \\
\hline Bifurcation buckling mode, $(m, n)$ & $(1,4)$ & $(1,4)$ & $(1,4)$ \\
\hline Limit-point load, lbs. & $1.07 \times 10^{7}$ & $1.06 \times 10^{7}$ & $1.06 \times 10^{7}$ \\
\hline
\end{tabular}




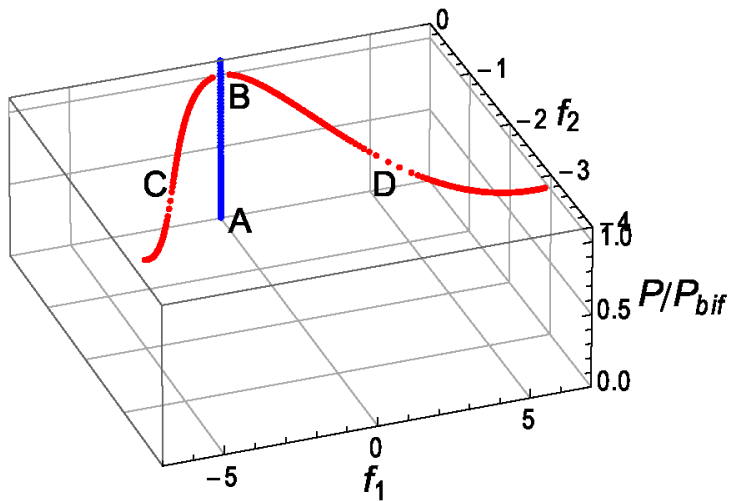

(a) Solution path, perfect

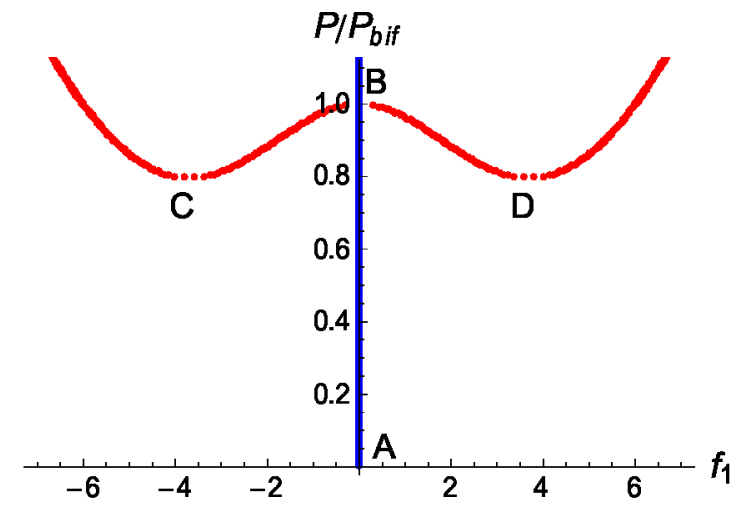

(c) Normalized load vs. $f_{1}$, perfect

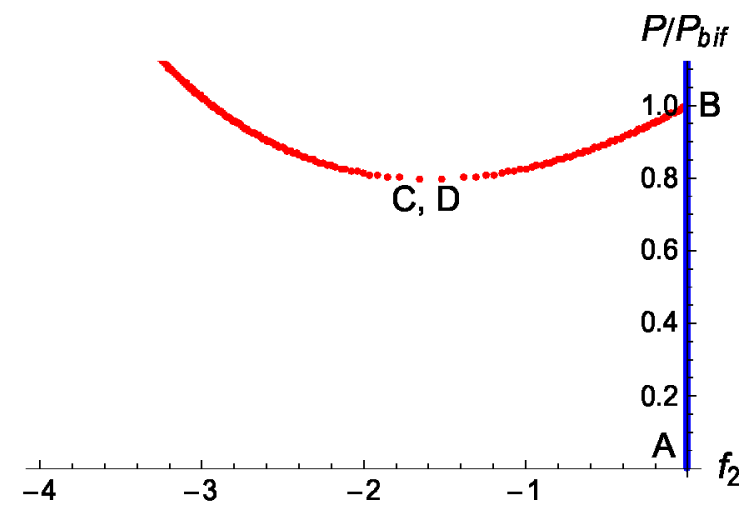

(e) Normalized load vs. $f_{2}$, perfect

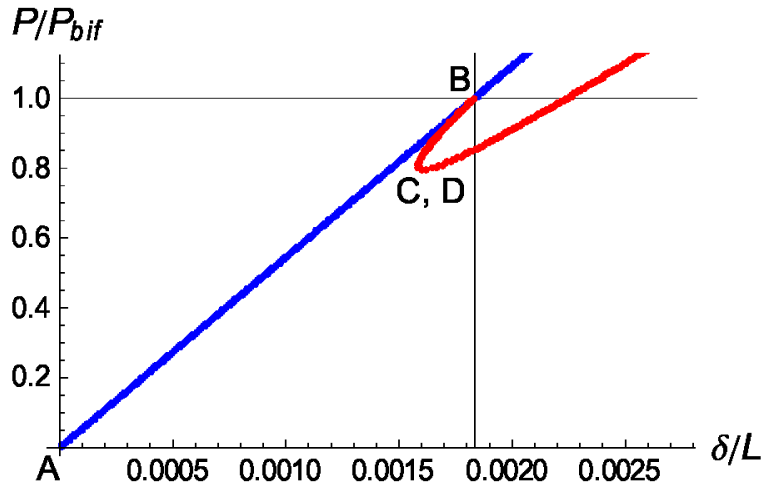

(g) Load-response curve, perfect

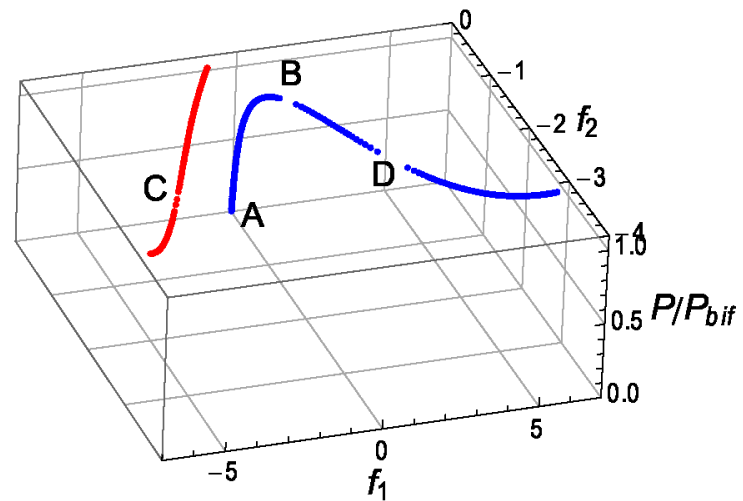

(b) Solution path, imperfect

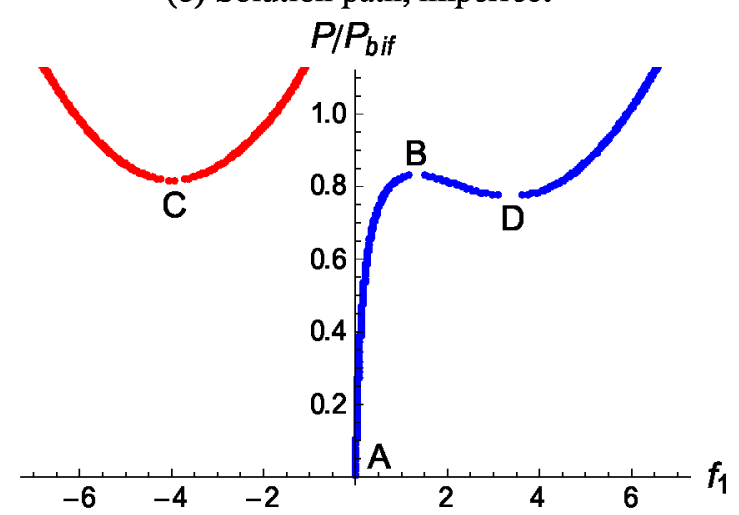

(d) Normalized load vs. $f_{1}$, imperfect

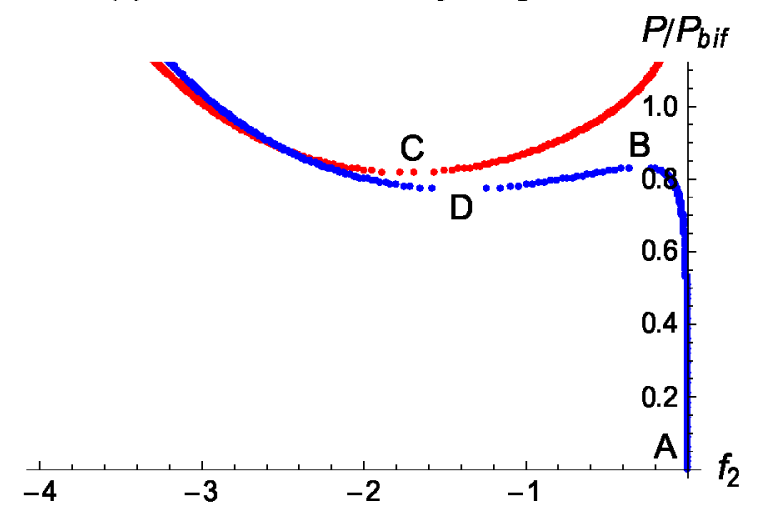

(f) Normalized load vs. $f_{2}$, imperfect

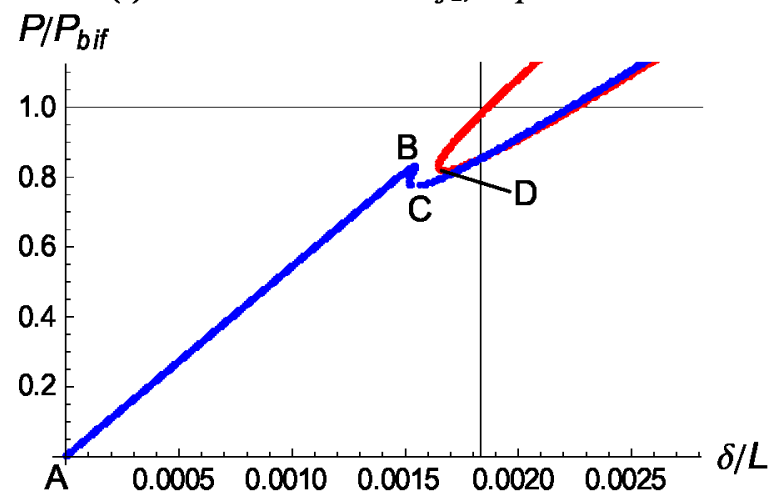

(h) Load-response curve, imperfect

Figure 3. Solution-path and load-response curves for mode $(m, n)=(9,15)$ of the isotropic cylinder. 


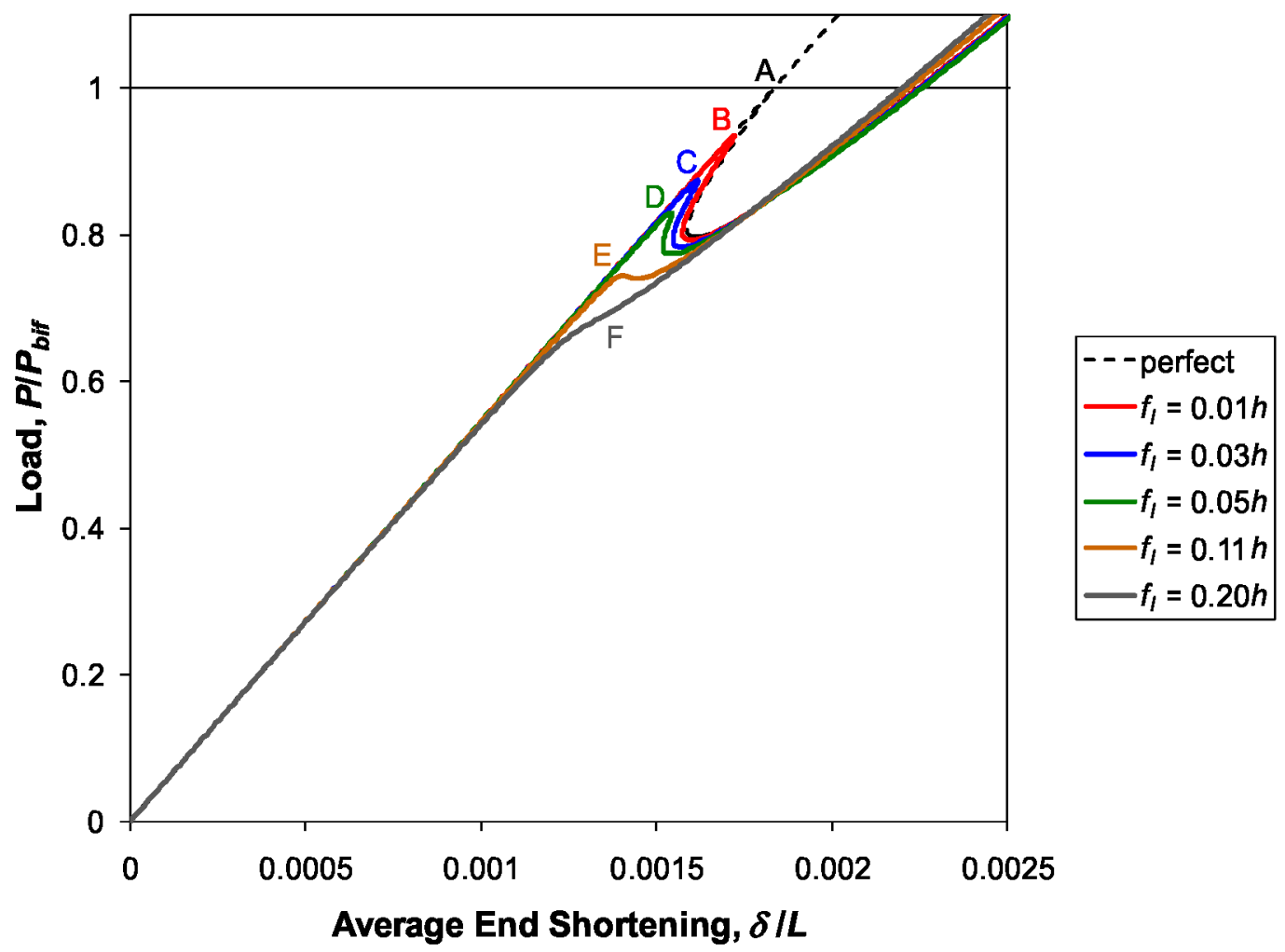

(a) Load-response curves



(b) Imperfection sensitivity factor as a function of imperfection amplitude

Figure 4. The effects of imperfection amplitude on the isotropic cylinder for mode $(m, n)=(9,15)$. 


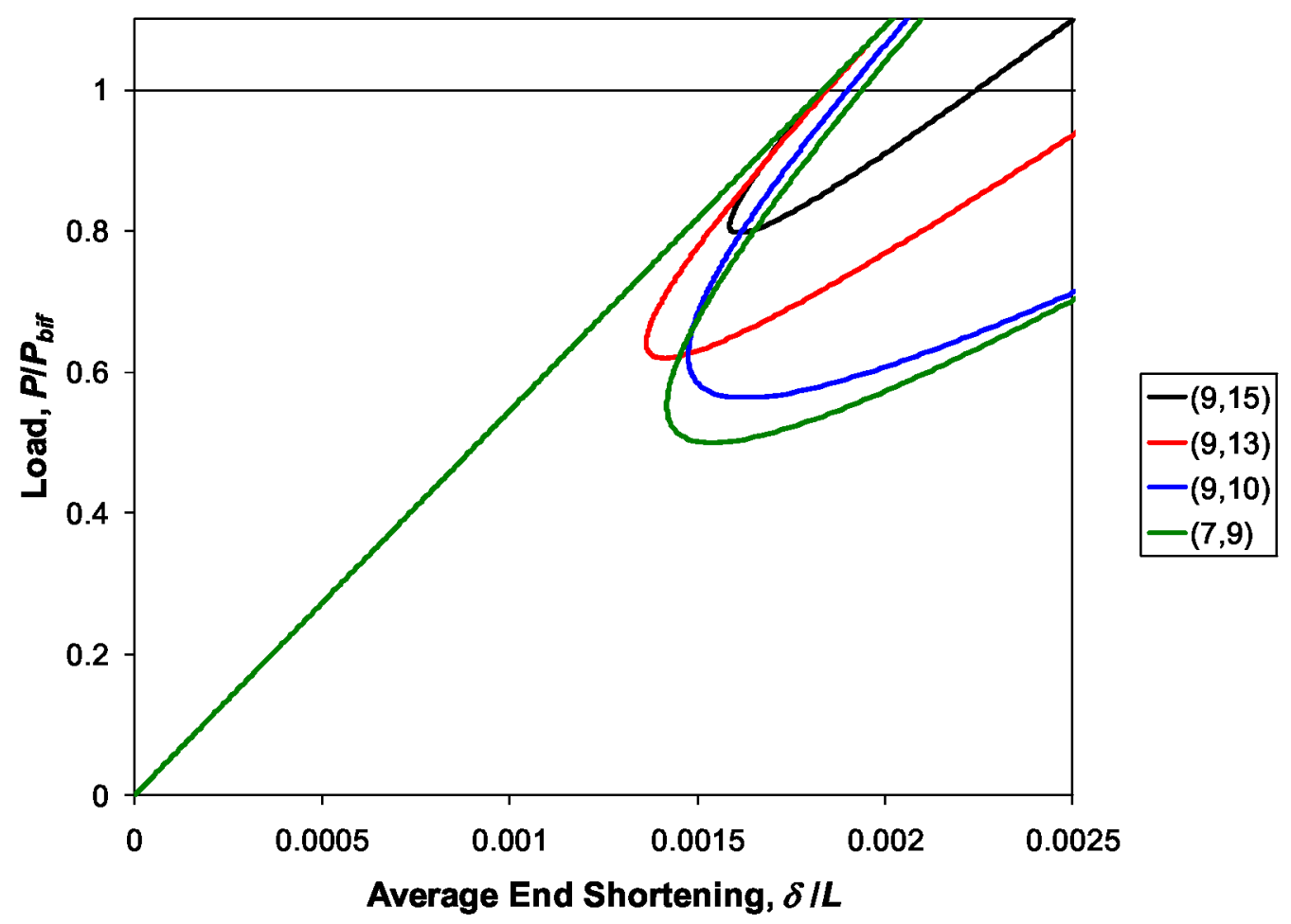

Figure 5. Load-response curves of the perfect isotropic cylinder for four modes: $(m, n)=(9,15),(9,13),(9,10)$, and $(7,9)$.

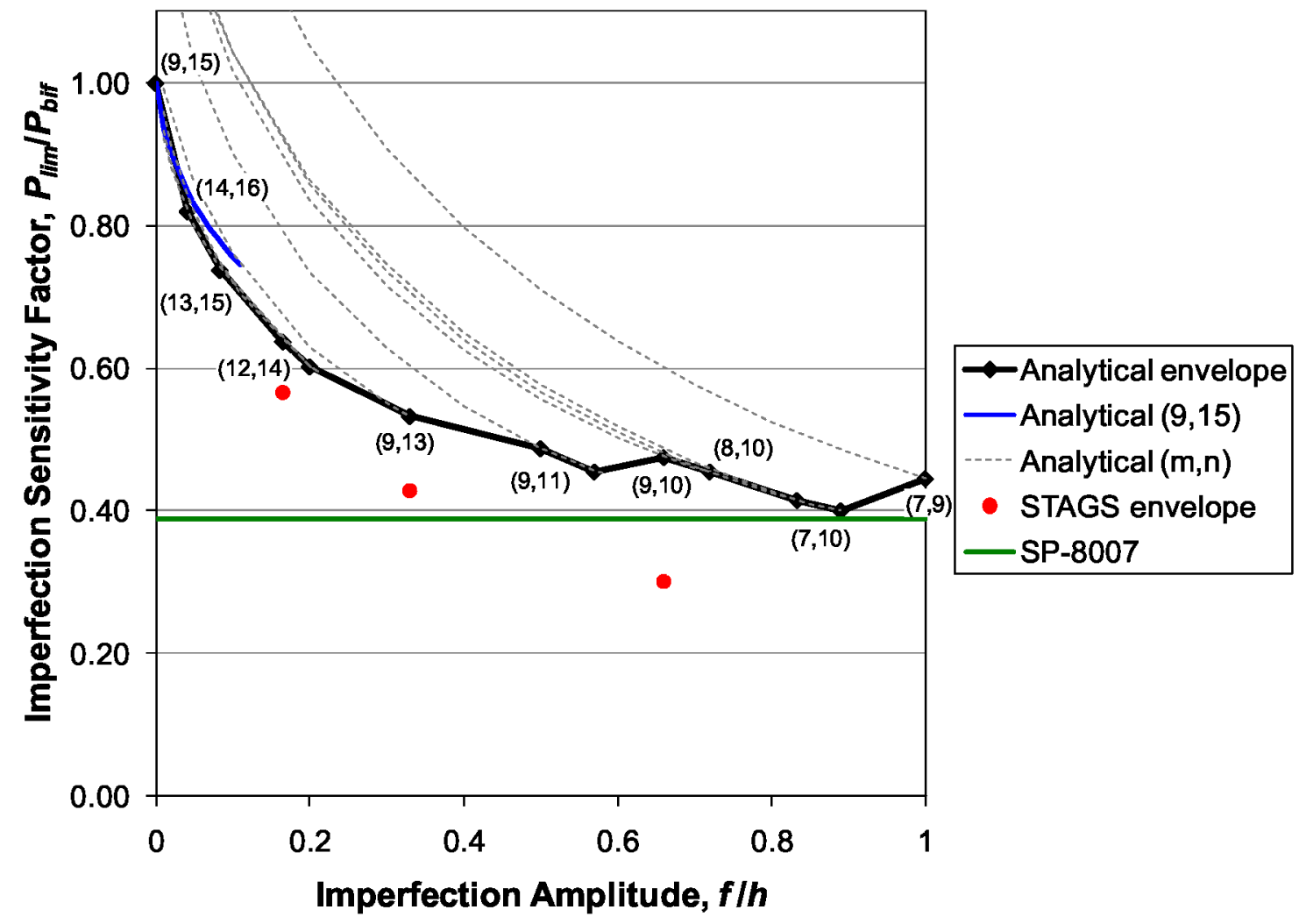

Figure 6. The envelope of imperfection sensitivity factors for a range of imperfection amplitudes.

American Institute of Aeronautics and Astronautics 


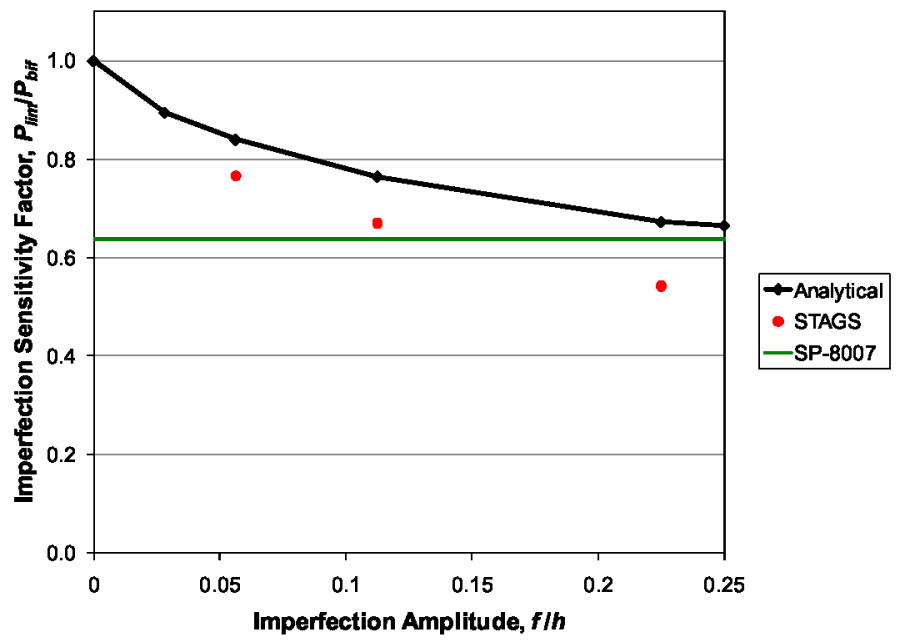

(a) Quasi-isotropic sandwich

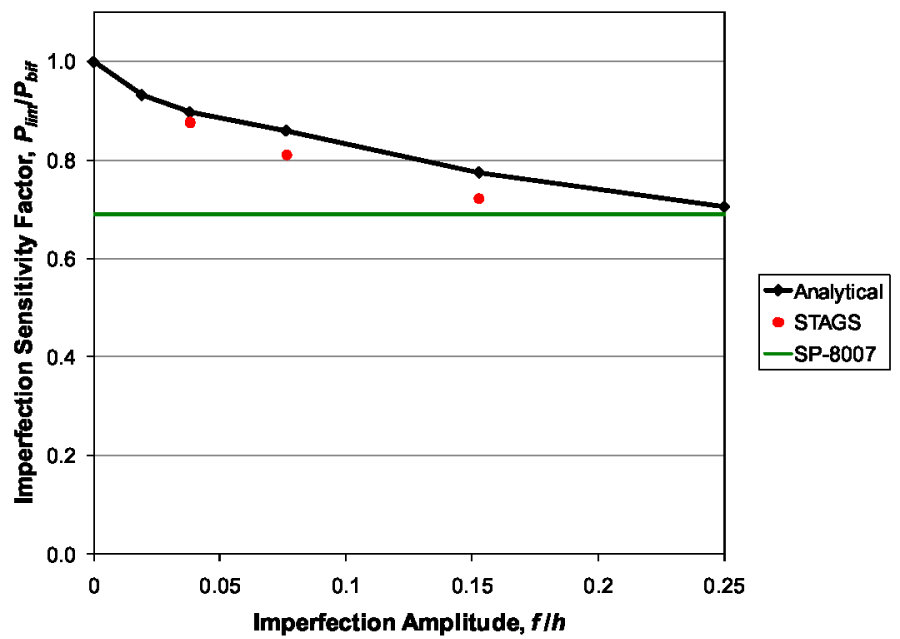

(b) Tailored sandwich

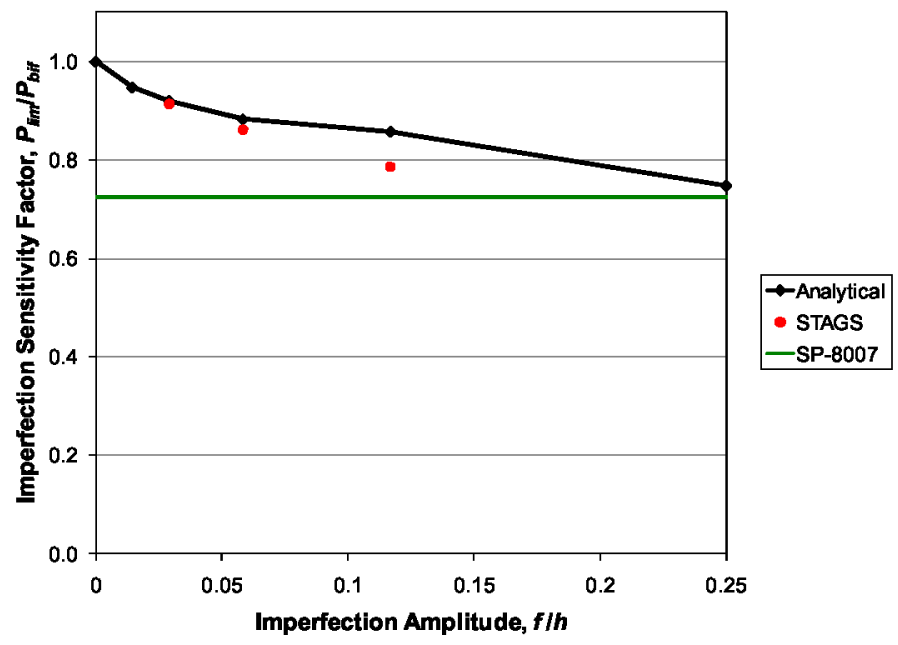

(c) Highly tailored sandwich

Figure 7. Imperfection sensitivity factors as a function of imperfection amplitude for the composite sandwich cylinders. 


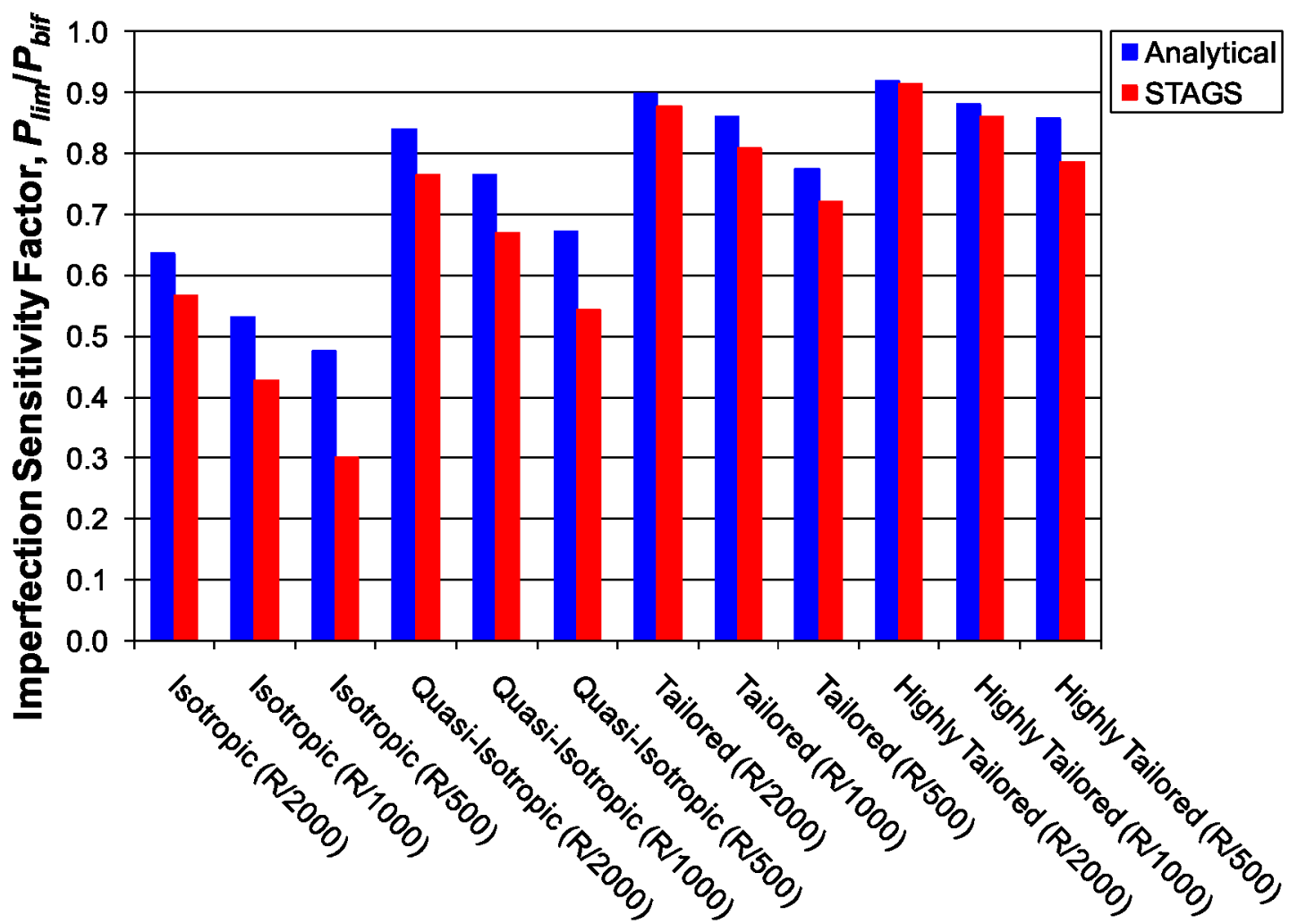

Figure 8. Imperfection knockdown factors from the analytical model and from STAGS for four shell constructions (isotropic, quasi-isotropic sandwich, tailored sandwich, and highly tailored sandwich) and three imperfection amplitudes $(R / 2000, R / 1000$, and $R / 500)$.

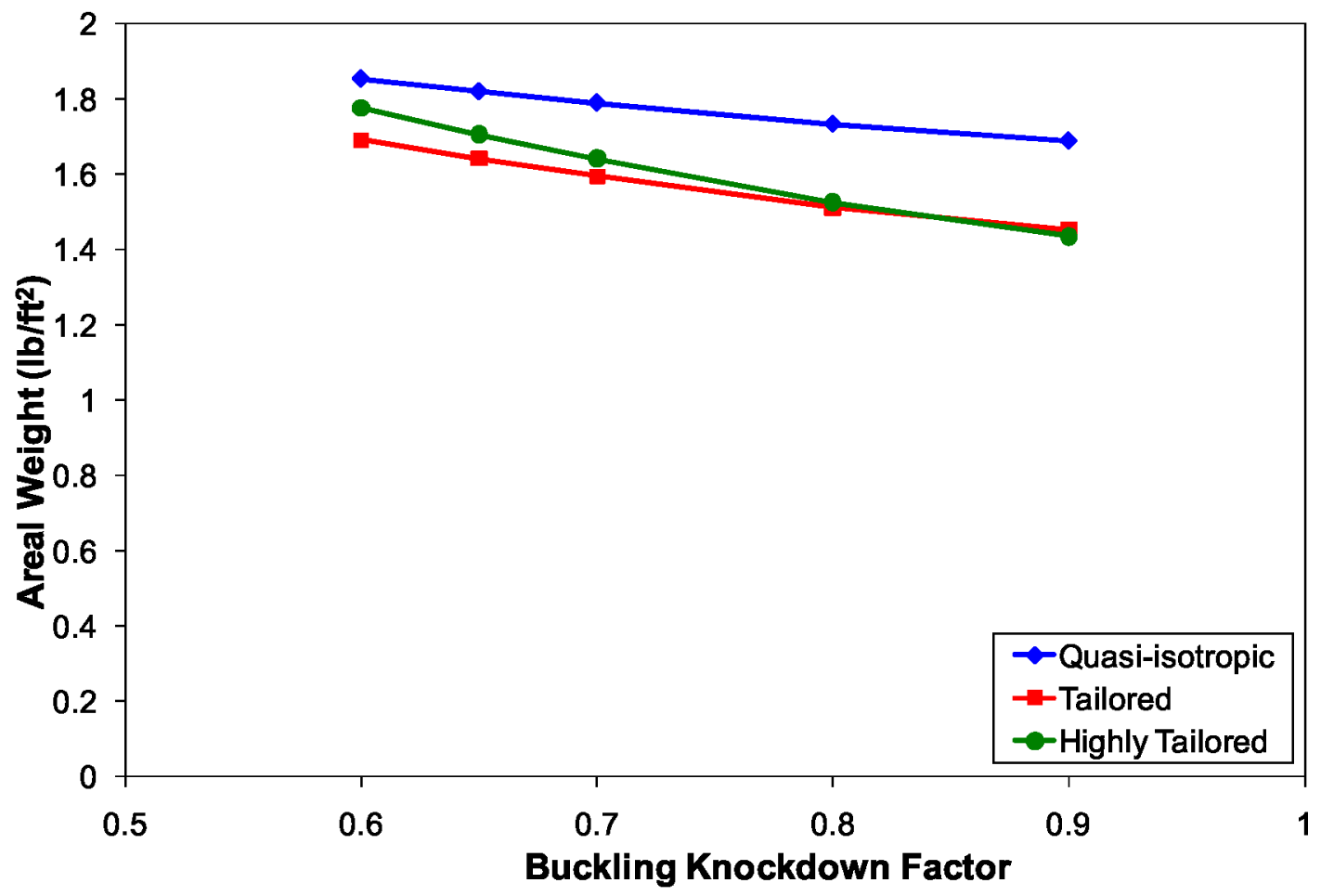

Figure 9. The effect of buckling knockdown factor on optimized areal weight. 